\title{
Well-posedness of the stochastic Boussinesq equation driven by Levy processes
}

\author{
Yan Zheng ${ }^{1}$ and Jianhua Huang ${ }^{1 *}$ (D)
}

\section{"Correspondence:}

jhhuang32@nudt.edu.cn

'Department of Mathematics,

National University of Defense

Technology, Changsha, P.R. China

\begin{abstract}
In this paper, we develop a new progressive stopping time technique to prove the existence and uniqueness of a special type of global solutions for the stochastic Boussinesq equations driven by Levy processes. Then we prove the existence of invariant measure.
\end{abstract}

MSC: $37\llcorner 05 ; 37\llcorner 40$

Keywords: Boussinesq equation; Levy process; Well-posedness; Invariant measure

\section{Introduction}

To model various phenomena in climate systems, geophysical, environmental, and so on, the Navier-Stokes equations may be coupled with other equations. Coupled with NavierStokes equations and the transport equations for temperature, the Boussinesq equation as a two-dimensional coupled system of Navier-Stokes equations and temperaturedependent transport equations can be used to describe the oceanic gravity currents $[3,9]$.

Recently there have been a few works related to the stochastic Boussinesq equation concentrating on various topics. For example, [13] and [18] are devoted to well-posedness problems while using different types of solution to deal with low regularity. [1, 19], and [15] study the random dynamics of the Boussinesq systems under diverse conditions on noise or random boundary and show the existence of random attractor. [10] considers the existence, uniqueness, and attraction properties of an ergodic invariant measure for the Boussinesq equations with degenerate Gaussian noise. [7, 16], and [20] progress gradually in the technical level and provide the large deviations principle for the Boussinesq equations with different random conditions. It is worth mentioning that all but a few works above are done under various Gaussian noises. Due to low regularity caused by noise or nonlinear terms, they may use weak, mild, or martingale solutions. However, we find that Levy noise with the characteristic of jump is really special in influencing the driven Boussinesq system, and the classical solution notion may not be appropriate, despite of [13] and [20], especially for further problems like ergodicity. So we adopt the original idea of [8] and put forward with "fragile solution".

To be specific, this article is concerned with the Boussinesq equations perturbed by Levy noise. The aim is to prove the existence and uniqueness of a special type of global solutions on the condition that Poisson measure possesses a $\sigma$-finite characteristic measure. Then we show that the related system possesses invariant measures. The novelty is that we

(c) The Author(s) 2019. This article is distributed under the terms of the Creative Commons Attribution 4.0 International License (http://creativecommons.org/licenses/by/4.0/), which permits unrestricted use, distribution, and reproduction in any medium, provided you give appropriate credit to the original author(s) and the source, provide a link to the Creative Commons license, and indicate if changes were made. 
adopt a special type of solution definition (fragile solution) to conquer low regularity, then we apply it successfully by obtaining well-posedness and studying statistically asymptotic behavior. To accomplish this, we develop a novel progressive stopping time technique to obtain the necessary a priori estimates. The critical point is that this method enables us to decompose the difficulties into several parts and tackle with the crucial regular problems. We believe that the current results can be generalized to other nonlocal models or the ones driven by more irregular noise. To illustrate this, in the last section we generalize the argument for stochastic Boussinesq equations to a class of abstract stochastic 2D hydrodynamical type systems driven by Levy noise with suitable modification. Roughly speaking, we can also obtain well-posedness for 2D magneto-hydrodynamic equation, 2D Boussinesq model for the Benard convection, 2D magnetic Benard problem, and 3D Leray $\alpha$ model for Navier-Stokes equations driven by Levy noise.

The rest of the paper is organized as follows. Section 2 is devoted to some preliminaries on notations, lemmas, and especially the definition of fragile solutions. In Sect. 3, we prove the existence and uniqueness of global fragile solution for the Boussinesq equation perturbed by Levy noise. In Sect. 4, we obtain the existence of equilibrium for the considered system. Finally, some discussions on the generalizations to abstract stochastic hydrodynamical equations driven by Levy noise are presented in Sect. 5 .

\section{Preliminary}

In the current paper, we study the stochastic Boussinesq equations driven by Levy white noises

$$
\left\{\begin{array}{l}
\frac{\partial u}{\partial t}+(u \cdot \nabla) u-v \Delta u+\nabla p=\theta e_{2}+Q_{1} d W_{1}(t)+\int_{U} f(u(t-), w) \tilde{N}_{1}(d t, d w), \\
\frac{\partial \theta}{\partial t}+(u \cdot \nabla) \theta-k \Delta \theta=u_{2}+Q_{2} d W_{2}(t)+\int_{U} g(\theta(t-), w) \tilde{N}_{2}(d t, d w), \\
\nabla \cdot u=0, \\
\left.u\right|_{\partial D}=0, \quad u(0)=u_{0}, \quad \theta(0)=\theta_{0},
\end{array}\right.
$$

with velocity $u=u(x, t)=\left(u_{1}, u_{2}\right) \in R^{2}$, salinity $\theta=\theta(x, t) \in R$, pressure $p, x=(\xi, \eta) \in D \subset$ $R^{2} . e_{2} \in R^{2}$ is a unit vector in the upward vertical direction. $Q_{1}$ and $Q_{2}$ are of trace class. $W_{1}(\cdot)\left(W_{2}(\cdot)\right.$, respectively) is an $\left(L^{2}(D)\right)^{2}\left(L^{2}(D)\right.$, respectively)-valued cylindrical Wiener process. $f$ ( $g$, respectively) is a measurable mapping from some measurable space $U$ to $\left(L^{2}(D)\right)^{2}\left(L^{2}(D)\right.$, respectively). $\tilde{N}_{1}$ and $\tilde{N}_{2}$ are compensated Poisson measures on $[0, \infty) \times U$ with intensity measures $\lambda_{1}$ an $\lambda_{2}$ being $\sigma$-finite measures on $\mathscr{B}(U)$. Assume that $W_{i}(t)$ and $\tilde{N}_{i}(d t, d u)$ are independent.

Let $L^{2}(D)$ be the Hilbert space. Denote $\mathbf{H}=\left(L^{2}(D)\right)^{2} \times L^{2}(D)$ with the scalar product and the induced norm

$$
(\phi, \psi)=\int_{D} \phi(x) \psi(x) d x, \quad|\phi|^{2}=(\phi, \phi), \quad \forall \phi \in \mathbf{H} .
$$

Set $\mathbf{V}=V_{1} \times V_{2}=\left(H^{1}(D)\right)^{2} \times H^{1}(D)$, then $\mathbf{V}$ is a product Hilbert space, of which the scalar product and the induced norm are given by

$$
(\phi, \psi)_{\mathbf{V}}=\int_{D} \nabla \phi \cdot \nabla \psi d x, \quad\|\phi\|_{\mathbf{V}}^{2}=(\phi, \phi)_{\mathbf{V}}=\left\|\phi_{1}\right\|_{V_{1}}^{2}+\left\|\phi_{2}\right\|_{V_{2}}^{2}
$$


By the classical interpolation inequality, there exists $C_{1}>0$ such that, for $u \in V_{1}, \theta \in V_{2}$,

$$
|u|_{\left(L^{4}(D)\right)^{2}}^{2} \leq C_{1}|u|\|u\|_{V_{1}}, \quad|\theta|_{L^{4}(D)}^{2} \leq C_{1}|\theta|\|\theta\|_{V_{2}} .
$$

Define an unbounded linear operator $A=\left(\nu A_{1}, k A_{2}\right): \mathbf{H} \rightarrow \mathbf{H}$ by

$$
\left(A_{1} u, v\right)=(u, u)_{V_{1}}, \quad\left(A_{2} \theta, \eta\right)=(\theta, \eta)_{V_{2}}, \quad \forall u, v \in D\left(A_{1}\right), \forall \theta, \eta \in D\left(A_{2}\right),
$$

where $D\left(A_{1}\right)=V_{1} \cap\left(H^{2}(D)\right)^{2}, D\left(A_{2}\right)=V_{2} \cap H^{2}(D)$, and $D(A)=D\left(A_{1}\right) \times D\left(A_{2}\right)$.

Introduce the following bilinear operators $B_{1}, B_{2}$ for every $u, v, w \in V_{1}, \theta, \eta \in V_{2}$ :

$$
\begin{aligned}
& \left(B_{1}(u, v), w\right)=\int_{D}[u \cdot \nabla v] w d x=\sum_{i, j=1,2} \int_{D} u_{i} \partial_{i} v_{j} w_{j} d x \\
& \left(B_{2}(u, \theta), \eta\right)=\int_{D}[u \cdot \nabla \theta] \eta d x=\sum_{i, j=1,2} \int_{D} u_{i} \partial_{i} \theta_{j} \eta_{j} d x .
\end{aligned}
$$

Denote

$$
\begin{aligned}
& A \phi=\left(\begin{array}{l}
v A_{1} u \\
k A_{2} \theta
\end{array}\right)=\left(\begin{array}{l}
-v \Delta u \\
-k \Delta \theta
\end{array}\right), \\
& B \phi=\left(\begin{array}{l}
B_{1}(u, u) \\
B_{2}(u, \theta)
\end{array}\right)=\left(\begin{array}{l}
(u \cdot \nabla) u \\
(u \cdot \nabla) \theta
\end{array}\right) .
\end{aligned}
$$

We will need some lemmas with regard to the properties of $A$ and $B$.

Lemma 2.1 ([1, Lemma 2.2]) $A$ is a positive, self-adjoint operator satisfying

$$
(A \phi, \phi) \geq \rho\|\phi\|_{\mathbf{H}}^{2}, \quad \rho=\min (v, k), \phi \in D(A) .
$$

Lemma 2.2 ([7, Lemma 3.4]) For $u, v, w \in V_{1}, \theta, \eta \in V_{2}$, there holds

(1) $\left(B_{1}(u, v), v\right)=0,\left(B_{2}(u, \theta), \theta\right)=0$,

(2) $\left(B_{1}(u, v), w\right)=-\left(B_{1}(u, w), v\right),\left(B_{2}(u, \theta), \eta\right)=-\left(B_{2}(u, \eta), \theta\right)$.

Lemma 2.3 ([7, Lemma 3.5]) For $u \in V_{1}, \theta, \eta \in V_{2}, \phi=(u, \theta)$, there holds

(1) $\left|B_{1}(u, v)\right|_{V_{1}^{\prime}} \leq|u|_{L^{4}}^{2} \leq C_{1}\|u\||u|$,

(2) $\left|\left(B_{2}(u, \theta), \eta\right)\right| \leq\|\eta\| \cdot|u|_{L^{4}} \cdot|\theta|_{L^{4}} \leq C_{1}\|\eta\| \cdot|u|^{\frac{1}{2}}\|u\|^{\frac{1}{2}} \cdot|\theta|^{\frac{1}{2}}\|\theta\|^{\frac{1}{2}}$.

To ease notation, set

$$
\begin{aligned}
& X=\left(\begin{array}{l}
u \\
\theta
\end{array}\right), \quad Q=\left(\begin{array}{l}
Q_{1} \\
Q_{2}
\end{array}\right), \quad W=\left(\begin{array}{l}
W_{1} \\
W_{2}
\end{array}\right) \\
& F(x, w)=\left(\begin{array}{c}
f(u, w) \\
g(\theta, w)
\end{array}\right), \quad R(X)=\left(\begin{array}{c}
-\theta e_{2} \\
-u_{2}
\end{array}\right), \\
& \tilde{N}(d t, d w)=\left(\begin{array}{c}
\tilde{N}_{1}(d t, d w) \\
\tilde{N}_{2}(d t, d w)
\end{array}\right), \quad \lambda=\left(\begin{array}{c}
\lambda_{1} \\
\lambda_{2}
\end{array}\right), \quad X(0)=x=\left(\begin{array}{c}
u_{0} \\
\theta_{0}
\end{array}\right) .
\end{aligned}
$$


Note that $\tilde{N}(d s, d w)$ is the compensated Poisson measure with intensity measure $\lambda$. We will consider the following equivalent abstract stochastic evolution equation in the sequel:

$$
\begin{aligned}
X_{t}= & x-\int_{0}^{t} A X_{s} d s-\int_{0}^{t} B\left(X_{s}\right) d s-\int_{0}^{t} R\left(X_{s}\right) d s \\
& +\int_{0}^{t} Q d W_{s}+\int_{0}^{t} \int_{U} F\left(X_{s-}, w\right) \tilde{N}(d s, d w) .
\end{aligned}
$$

Due to the low regularity of the noise, we cannot expect that the solution $X$ to equation (2.3) is square integrable in time with values in $\mathbf{V}$. So we introduce the following unconventional definition.

Definition 2.1 A fragile solution to equation (2.3) is a progressively measurable stochastic process $X_{t}$ in $[0, T]$ with

$$
X(\omega) \in C([0, T] ; \mathbf{H}) \cap L^{2}\left(0, T ; \mathscr{D}\left(A^{\frac{1}{4}}\right)\right)
$$

for a.e. $\omega \in \Omega$, such that

$$
\begin{gathered}
\left(X_{t}, \phi\right)+\int_{0}^{t}\left(X_{s}, A \phi\right) d s+\int_{0}^{t}\left(B\left(X_{s}, \phi\right), X_{s}\right) d s+\int_{0}^{t}\left(R\left(X_{s}, \phi\right), X_{s}\right) d s \\
=(x, \phi)+\int_{0}^{t} \int_{U}\left(F\left(X_{s-}, u\right), \phi\right) \tilde{N}(d s, d u)+\left(W_{t}, Q \phi\right)
\end{gathered}
$$

a.e. for all $t \in[0, T]$ and all $\phi \in \mathscr{D}(A)$.

Note that this definition coincides with the generalized solution introduced by [8]. Here we adopt the name "fragile" to distinguish from "weak".

Now we impose some hypotheses on $F$, which are also included in [5]. Suppose that $\left\{U_{k}\right\}_{k \geq 1}$ are the measurable subsets of $U$ on the condition that $U_{k} \nearrow U$ and $\lambda\left(U_{k}\right)<\infty$. There exist positive constants $C$ and $K$ such that, for some $\alpha \in[1 / 4,1 / 2)$,

$\left(\mathrm{H}_{1}\right) Q: \mathbf{H} \rightarrow \mathbf{H}$ is a linear bounded operator, whose range $\mathscr{R}(Q)$ is dense in $\mathscr{D}\left(A^{\frac{1}{4}+\frac{\alpha}{2}}\right)$ and $\mathscr{D}\left(A^{2 \alpha}\right) \subset \mathscr{R}(Q) \subset \mathscr{D}\left(A^{\frac{1}{4}+\frac{\alpha}{2}+\varepsilon}\right)$ for some $\varepsilon>0$;

$\left(\mathrm{H}_{2}\right) \int_{U}\left|A^{\alpha} F(0, u)\right|^{2} \lambda(d u)=C$;

$\left(\mathrm{H}_{3}\right) \int_{U}\left|A^{\alpha}(F(x, u)-F(y, u))\right|^{2} \lambda(d u) \leq K\left|A^{\alpha}(x-y)\right|^{2}$;

$\left(\mathrm{H}_{4}\right) \sup _{x \in H} \int_{U_{m}^{c}}\left|A^{\alpha} F(x, u)\right|^{2} \lambda(d u) \rightarrow 0$, as $m \rightarrow \infty$.

\section{Global solutions}

\section{$3.1 \lambda(U)<\infty$}

Throughout this subsection we suppose that $\lambda(U)<\infty$. Noting that the character measure $\lambda(U)$ is finite, we will rearrange the jump times of $N(d t, d u)$. Let the jump times of $N(d t, d u)$ be $\sigma_{1}(\omega)<\sigma_{2}(\omega)<\cdots$, then on $\left[0, \sigma_{1}\right)$, equation (2.3) can be regarded as the equivalence of the following deterministic integral equation:

$$
\begin{aligned}
X_{t}= & x-\int_{0}^{t} A X_{s} d s-\int_{0}^{t} B\left(X_{s}\right) d s-\int_{0}^{t} R\left(X_{s}\right) d s \\
& +\int_{0}^{t} Q d W_{s}+\int_{0}^{t} \int_{U} F\left(X_{s-}, z\right) \lambda(d s, d z) .
\end{aligned}
$$


It is well known that the Ornstein-Uhlenbeck process $z$ is the solution of

$$
\left\{\begin{array}{l}
d z_{t}+A z_{t} d t=Q d W_{t} \\
z_{0}=0
\end{array}\right.
$$

Lemma 3.1 If hypothesis $\left(\mathrm{H}_{1}\right)$ holds, then $z \in C_{0}\left([0, T], \mathscr{D}\left(A^{1 / 4+\alpha / 2}\right)\right)$ a.s.

Proof Since $A^{\frac{1}{4}+\frac{\alpha}{2}+\varepsilon}$ is continuous in $\mathbf{H}$ by the closed graph theorem and $A^{-\frac{1}{2}-\frac{\varepsilon}{2}}$ is HilbertSchmidt in $\mathbf{H}, A^{-\frac{1}{4}+\frac{\alpha}{2}+\frac{\varepsilon}{2}} Q=A^{-\frac{1}{2}-\frac{\varepsilon}{2}}\left(A^{\frac{1}{4}+\frac{\alpha}{2}+\varepsilon} Q\right)$ is a Hilbert-Schmidt operator on $\mathbf{H}$. Let $e_{k}$, $k \in \mathbf{N}$ be the normalized eigenfunctions corresponding to the eigenvalues $0<\lambda_{1} \leq \lambda_{2} \leq$ $\cdots$ of $A$. Letting $Q e_{n}=\sum_{k=1}^{\infty} q_{n k} e_{k}$ and $\sigma_{k}^{2}=\sum_{n=1}^{\infty} q_{n k}^{2}$, one finds that

$$
\sum_{k=1}^{\infty} \sigma_{k}^{2} \lambda_{k}^{-\frac{1}{2}+\alpha+\varepsilon}=\sum_{k=1}^{\infty} \sum_{n=1}^{\infty} q_{n k}^{2} \lambda_{k}^{-\frac{1}{2}+\alpha+\varepsilon}=\left|A^{-\frac{1}{4}+\frac{\alpha}{2}+\varepsilon} Q\right|_{\mathrm{HS}}<\infty
$$

With this inequality, one can proceed similarly as [8, p. 411] did to obtain

$$
E\left|z_{t}\right|_{\mathscr{D}\left(A^{\left.\frac{1}{4}+\frac{\alpha}{2}+\varepsilon_{0}\right)}\right.}^{4} \leq 2\left(\sum_{n=1}^{\infty} \sigma_{n}^{2} \lambda_{n}^{-\frac{1}{2}+\alpha+2 \varepsilon_{0}}\right)^{2}
$$

for some $\varepsilon_{0}<\frac{1}{2} \varepsilon$.

By the change of variable $Y_{t}=X_{t}-z_{t}$, we transform equation (2.3) into

$$
\begin{aligned}
Y_{t}= & x-\int_{0}^{t} A Y_{s} d s-\int_{0}^{t} B\left(Y_{s}+z_{s}\right) d s-\int_{0}^{t} R\left(Y_{s}+z_{s}\right) d s \\
& +\int_{0}^{t} \int_{U} F\left(X_{s}+z_{s}, z\right) \lambda(d z, d t) .
\end{aligned}
$$

Theorem 3.1 If hypotheses $\left(\mathrm{H}_{1}\right)-\left(\mathrm{H}_{3}\right)$ hold, then for $\forall x \in \mathscr{D}\left(A^{\alpha}\right)$, there exists a unique solution $X$ of (3.1) such that, for a.s. $\omega \in \Omega$,

$$
X .(\omega)-z .(\omega) \in C\left([0, T], \mathscr{D}\left(A^{\alpha}\right)\right) \cap L^{\frac{4}{1-2 \alpha}}\left(0, T ; \mathscr{D}\left(A^{\frac{1}{4}+\frac{\alpha}{2}}\right)\right) \cap L^{2}\left(0, T ; \mathscr{D}\left(A^{\frac{1}{2}+\alpha}\right)\right) .
$$

The transition semigroup associated with (3.2) is a Feller Markov process.

Proof Our strategy is to utilize the Galerkin method. Denote $\mathbf{E}_{n}=\operatorname{span}\left\{e_{1}, e_{2}, \ldots, e_{n}\right\}$, $P_{n}: \mathbf{H} \rightarrow \mathbf{E}_{n}$ is an orthonormal projection. Consider the following ordinary differential equation:

$$
\left\{\begin{array}{l}
\frac{d Y_{t}^{n}}{d t}=-A Y_{s}^{n}-P_{n} B\left(Y_{s}^{n}+z_{s}^{n}\right)-P_{n} R\left(Y_{s}^{n}+z_{s}^{n}\right)+\int_{U} F\left(Y_{s}^{n}+z_{s}^{n}, u\right) \lambda(d u) \\
Y_{0}^{n}=P_{n} x
\end{array}\right.
$$

where $z_{t}^{n}$ is the Ornstein-Uhlenbeck process satisfying

$$
\left\{\begin{array}{l}
d z_{t}^{n}+A z_{t}^{n} d t=P_{n} Q d W_{t} \\
z_{0}^{n}=0
\end{array}\right.
$$


Multiplying equation (3.3) by $Y_{t}^{n}$ yields

$$
\begin{aligned}
\frac{1}{2} & \frac{d}{d t}\left|Y_{t}^{n}\right|^{2}+\left\|Y_{t}^{n}\right\|^{2} \\
= & -\left(P_{n} B\left(Y_{t}^{n}+z_{t}^{n}\right), Y_{t}^{n}\right)-\left(P_{n} R\left(Y_{t}^{n}+z_{t}^{n}\right), Y_{t}^{n}\right)+\int_{U}\left(F\left(Y_{s}^{n}+z_{s}^{n}, u\right), Y_{t}^{n}\right) \lambda(d u) \\
\leq & \left|\left(B\left(Y_{t}^{n}, Y_{t}^{n}\right), z_{t}^{n}\right)\right|+\left|\left(B\left(z_{t}^{n}, Y_{t}^{n}\right), z_{t}^{n}\right)\right|+\left|Y_{t}^{n}+z_{t}^{n}\right|\left|Y_{t}^{n}\right|+\int_{U}\left(F\left(Y_{s}^{n}+z_{s}^{n}, u\right), Y_{t}^{n}\right) \lambda(d u) \\
\leq & \frac{1}{2}\left\|Y_{t}^{n}\right\|^{2}+C_{1}\left|z_{t}^{n}\right|_{L^{4}}^{4}\left|Y_{t}^{n}\right|^{2}+C_{1}\left|z_{t}^{n}\right|_{L^{4}}^{4}+\left|Y_{t}^{n}+z_{t}^{n}\right|\left|Y_{t}^{n}\right| \\
& +\int_{U}\left|Y_{t}^{n}\right|\left|F\left(Y_{s}^{n}+z_{s}^{n}, u\right)-F(0, u)+F(0, u)\right| \lambda(d u) \\
\leq & \frac{1}{2}\left\|Y_{t}^{n}\right\|^{2}+C_{1}\left|z_{t}^{n}\right|_{L^{4}}^{4}\left|Y_{t}^{n}\right|^{2}+C_{1}\left|z_{t}^{n}\right|_{L^{4}}^{4}+\left|Y_{t}^{n}+z_{t}^{n}\right|\left|Y_{t}^{n}\right|+\lambda(U)\left|Y_{t}^{n}\right|^{2} \\
& +\frac{2 K}{\lambda_{1}^{\alpha}}\left[\left|Y_{t}^{n}\right|^{2}+\left|z_{t}^{n}\right|^{2}\right]+\frac{C}{\lambda_{1}^{\alpha}} \\
\leq & \frac{1}{2}\left\|Y_{t}^{n}\right\|^{2}+\left[2+\lambda(U)+C_{1}\left|z_{t}^{n}\right|_{L^{4}}^{4}+\frac{2 K}{\lambda_{1}^{\alpha}}\right]\left|Y_{t}^{n}\right|^{2}+\left(1+\frac{2 K}{\lambda_{1}^{\alpha}}\right)\left|z_{t}^{n}\right|^{2} \\
& +C_{1}\left|z_{t}^{n}\right|_{L^{4}}^{4}+\frac{C}{\lambda_{1}^{\alpha}},
\end{aligned}
$$

where $C_{1}$ is a positive constant, whose value may change from one line to another. By the way, we will abuse notation in this way in the sequel. By Gronwall's inequality, we get

$$
\begin{aligned}
& \sup _{0 \leq t \leq T}\left|Y_{t}^{n}\right|^{2}+\int_{0}^{T}\left\|Y_{t}^{n}\right\|^{2} d t \\
& \leq|x|^{2} e^{\int_{0}^{T}\left[4+2 \lambda(U)+2 C_{1}\left|z_{t}^{n}\right|_{L^{4}}^{4}+\frac{4 K}{\lambda_{1}^{\alpha}}\right] d t} \\
& \quad+\int_{0}^{T} e^{\int_{0}^{t}\left[4+2 \lambda(U)+2 C_{1}\left|z_{t}^{n}\right|_{L^{4}}^{4}+\frac{4 K}{\lambda_{1}^{\alpha}}\right] d s}\left[\left(2+\frac{4 K}{\lambda_{1}^{\alpha}}\right)\left|z_{t}^{n}\right|^{2}+2 C_{1}\left|z_{t}^{n}\right|_{L^{4}}^{4}+\frac{2 C}{\lambda_{1}^{\alpha}}\right] d t .
\end{aligned}
$$

From this we conclude that $\left\{Y_{t}^{n}\right\}_{n \geq 1}$ is bounded in $L^{\infty}(0, T ; \mathbf{H}) \cap L^{2}(0, T ; \mathbf{V})$, because $z_{t}^{n}$ has continuous trajectories with values in $\mathscr{D}\left(A^{\frac{1}{4}}\right) \subset\left[L^{4}(\mathscr{O})\right]^{2}$. Consequently, $Y_{t}^{n}$ cannot explode in finite time.

Multiplying equation (3.3) by $A^{2 \alpha} Y_{t}^{n}$ and applying the interpolation inequality

$$
\left|A^{\frac{1}{4}+\frac{\alpha}{2}} Y_{t}^{n}\right|^{2} \leq C_{1}\left|A^{\alpha} Y_{t}^{n}\right|\left|A^{\frac{1}{2}} Y_{t}^{n}\right|
$$

we have

$$
\begin{aligned}
\frac{1}{2} & \frac{d}{d t}\left|A^{\alpha} Y_{t}^{n}\right|^{2}+\left|A^{\frac{1}{2}+\alpha} Y_{t}^{n}\right|^{2} \\
= & -\left(P_{n} B\left(Y_{t}^{n}+z_{t}^{n}\right), A^{2 \alpha} Y_{t}^{n}\right)-\left(A^{\alpha} P_{n} R\left(Y_{t}^{n}+z_{t}^{n}\right), A^{\alpha} Y_{t}^{n}\right) \\
& \quad+\int_{U}\left(A^{\alpha} F\left(Y_{s}^{n}+z_{s}^{n}, u\right), A^{\alpha} Y_{t}^{n}\right) \lambda(d u) \\
\leq & C_{1}\left|A^{\frac{1}{4}+\frac{\alpha}{2}}\left(Y_{t}^{n}+z_{t}^{n}\right)\right|^{2}\left|A^{\alpha+\frac{1}{2}} Y_{t}^{n}\right|+\left|\left(A^{\alpha} P_{n} R\left(Y_{t}^{n}+z_{t}^{n}\right), A^{\alpha} Y_{t}^{n}\right)\right|
\end{aligned}
$$




$$
\begin{aligned}
& +\left|\int_{U}\left(A^{\alpha} F\left(Y_{s}^{n}+z_{s}^{n}, u\right), A^{\alpha} Y_{t}^{n}\right) \lambda(d u)\right| \\
\leq & C_{1}\left|A^{\frac{1}{4}+\frac{\alpha}{2}}\left(Y_{t}^{n}+z_{t}^{n}\right)\right|^{2}\left|A^{\alpha+\frac{1}{2}} Y_{t}^{n}\right|+\left|A^{\alpha}\left(Y_{t}^{n}+z_{t}^{n}\right)\right|\left|A^{\alpha} Y_{t}^{n}\right|+\lambda(U)\left|A^{\alpha} Y_{t}^{n}\right|^{2} \\
& +2 K\left[\left|A^{\alpha} Y_{t}^{n}\right|^{2}+\left|A^{\alpha} z_{t}^{n}\right|^{2}\right]+C \\
\leq & 2 C_{1}\left|A^{\alpha} Y_{t}^{n}\right|\left|A^{\frac{1}{2}} Y_{t}^{n}\right|\left|A^{\alpha+\frac{1}{2}} Y_{t}^{n}\right|+2 C_{1}\left|A^{\frac{1}{4}+\frac{\alpha}{2}} z_{t}^{n}\right|^{2}\left|A^{\alpha+\frac{1}{2}} Y_{t}^{n}\right| \\
& +(2+\lambda(U)+2 K)\left|A^{\alpha} Y_{t}^{n}\right|^{2}+(2 K+1)\left|A^{\alpha} z_{t}^{n}\right|^{2}+C \\
\leq & \frac{1}{2}\left|A^{\frac{1}{2}+\alpha} Y_{t}^{n}\right|^{2}+C_{2}\left|A^{\frac{1}{2}} Y_{t}^{n}\right|^{2}\left|A^{\alpha} Y_{t}^{n}\right|^{2}+C_{2}\left|A^{\frac{1}{4}+\frac{\alpha}{2}} z_{t}^{n}\right|^{4}+(2+\lambda(U)+2 K)\left|A^{\alpha} Y_{t}^{n}\right|^{2} \\
& +(2 K+1)\left|A^{\alpha} z_{t}^{n}\right|^{2}+C \\
\leq & \frac{1}{2}\left|A^{\frac{1}{2}+\alpha} Y_{t}^{n}\right|^{2}+\left(2+\lambda(U)+2 K+C_{2}\left|A^{\frac{1}{2}} Y_{t}^{n}\right|^{2}\right)\left|A^{\alpha} Y_{t}^{n}\right|^{2}+C_{2}\left|A^{\frac{1}{4}+\frac{\alpha}{2}} z_{t}^{n}\right|^{4} \\
& +(2 K+1)\left|A^{\alpha} z_{t}^{n}\right|^{2}+C .
\end{aligned}
$$

Gronwall's inequality implies

$$
\begin{aligned}
& \sup _{0 \leq t \leq T}\left|A^{\alpha} Y_{t}^{n}\right|^{2}+\int_{0}^{T}\left|A^{\frac{1}{2}+\alpha} Y_{t}^{n}\right|^{2} d t \\
& \leq\left|A^{\alpha} x\right|^{2} e^{\int_{0}^{T}\left[4+2 \lambda(U)+4 K+2 C_{2}\left|A^{\frac{1}{2}} Y_{t}^{n}\right|^{2}\right] d t}+\int_{0}^{T} e^{\int_{0}^{t}\left[4+2 \lambda(U)+4 K+2 C_{2}\left|A^{\frac{1}{2}} Y_{s}^{n}\right|^{2}\right] d s}\left[2 C_{2}\left|A^{\frac{1}{4}+\frac{\alpha}{2}} z_{t}^{n}\right|^{4}\right. \\
& \left.\quad+2(2 K+1)\left|A^{\alpha} z_{t}^{n}\right|^{2}+2 C\right] d t .
\end{aligned}
$$

Since $z_{t}^{n}$ has continuous trajectories with values in $\mathscr{D}\left(A^{\frac{1}{4}+\frac{\alpha}{2}}\right) \subset \mathscr{D}\left(A^{\alpha}\right)$, we derive from this and (3.4) that $\left\{Y_{t}^{n}\right\}_{n \geq 1}$ is bounded in $L^{\infty}\left(0, T ; \mathscr{D}\left(A^{\alpha}\right)\right) \cap L^{2}\left(0, T ; \mathscr{D}\left(A^{\frac{1}{2}+\alpha}\right)\right)$. Noting the interpolation inequality

$$
\left|A^{\frac{1}{4}+\frac{\alpha}{2}} Y_{t}^{n}\right| \leq C_{1}\left|A^{\alpha} Y_{t}^{n}\right|^{\frac{1}{2}+\alpha}\left|A^{\frac{1}{2}+\alpha} Y_{t}^{n}\right|^{\frac{1}{2}-\alpha},
$$

we get $Y \in L^{\frac{4}{1-2 \alpha}}\left(0, T ; \mathscr{D}\left(A^{\frac{1}{4}+\frac{\alpha}{2}}\right)\right)$. Utilizing weakly and weakly $*$-convergent subsequences and noting that $z^{n}$ is strongly convergent to $z$, one obtains the existence of a solution $Y \in$ $L^{\infty}\left(0, T ; \mathscr{D}\left(A^{\alpha}\right)\right) \cap L^{2}\left(0, T ; \mathscr{D}\left(A^{\frac{1}{2}+\alpha}\right)\right) \cap L^{\frac{4}{1-2 \alpha}}\left(0, T ; \mathscr{D}\left(A^{\frac{1}{4}+\frac{\alpha}{2}}\right)\right)$. By the classical argument (cf. [17, Chap. 3]), we get $Y \in C\left(0, T ; \mathscr{D}\left(A^{\alpha}\right)\right)$.

Finally, we examine the uniqueness. Suppose that $Y^{(1)}$ and $Y^{(2)}$ are two solutions, then

$$
\begin{aligned}
& \frac{d\left(Y_{t}^{(1)}-Y_{t}^{(2)}\right)}{d t}+A\left(Y_{t}^{(1)}-Y_{t}^{(2)}\right)+B\left(Y_{t}^{(1)}-Y_{t}^{(2)}, Y_{t}^{(1)}+z_{t}\right)+B\left(Y_{t}^{(2)}+z_{t}, Y_{t}^{(1)}-Y_{t}^{(2)}\right) \\
& \quad+R\left(Y_{t}^{(1)}-Y_{t}^{(2)}, Y_{t}^{(1)}+z_{t}\right)+R\left(Y_{t}^{(2)}+z_{t}, Y_{t}^{(1)}-Y_{t}^{(2)}\right) \\
& =\int_{U}\left[F\left(Y_{t}^{(2)}+z_{t}, u\right)-F\left(Y_{t}^{(1)}+z_{t}, u\right)\right] \lambda(d u) .
\end{aligned}
$$

Therefore, by Lemma 2.3,

$$
\begin{aligned}
& \frac{1}{2} \frac{d\left|Y_{t}^{(1)}-Y_{t}^{(2)}\right|^{2}}{d t}+\left\|Y_{t}^{(1)}-Y_{t}^{(2)}\right\|^{2} \\
& \quad \leq\left|\left(B\left(Y_{t}^{(1)}-Y_{t}^{(2)}, Y_{t}^{(1)}+z_{t}\right), Y_{t}^{(1)}-Y_{t}^{(2)}\right)\right|+\left|\left(B\left(Y_{t}^{(2)}+z_{t}, Y_{t}^{(1)}-Y_{t}^{(2)}\right), Y_{t}^{(1)}-Y_{t}^{(2)}\right)\right|
\end{aligned}
$$




$$
\begin{aligned}
& +\left|\left(R\left(Y_{t}^{(1)}-Y_{t}^{(2)}, Y_{t}^{(1)}+z_{t}\right), Y_{t}^{(1)}-Y_{t}^{(2)}\right)\right|+\left|\left(R\left(Y_{t}^{(2)}+z_{t}, Y_{t}^{(1)}-Y_{t}^{(2)}\right), Y_{t}^{(1)}-Y_{t}^{(2)}\right)\right| \\
& +\int_{U}\left|\left(F\left(Y_{t}^{(2)}+z_{t}, u\right)-F\left(Y_{t}^{(1)}+z_{t}, u\right), Y_{t}^{(1)}-Y_{t}^{(2)}\right)\right| \lambda(d u) \\
\leq & C_{1}\left\|Y_{t}^{(1)}-Y_{t}^{(2)}\right\|\left|Y_{t}^{(1)}-Y_{t}^{(2)}\right|_{L^{4}}\left|Y_{t}^{(1)}+z_{t}\right|_{L^{4}} \\
& +C_{1}\left\|Y_{t}^{(1)}-Y_{t}^{(2)}\right\|\left|Y_{t}^{(1)}-Y_{t}^{(2)}\right|_{L^{4}}\left|Y_{t}^{(2)}+z_{t}\right|_{L^{4}} \\
& +\left|Y_{t}^{(1)}-Y_{t}^{(2)}\right| \cdot\left|Y_{t}^{(1)}+z_{t}\right| \cdot\left|Y_{t}^{(1)}-Y_{t}^{(2)}\right|+\left|Y_{t}^{(1)}-Y_{t}^{(2)}\right| \cdot\left|Y_{t}^{(2)}+z_{t}\right| \cdot\left|Y_{t}^{(1)}-Y_{t}^{(2)}\right| \\
& +\int_{U}\left|F\left(Y_{t}^{(2)}+z_{t}, u\right)-F\left(Y_{t}^{(1)}+z_{t}, u\right)\right| \cdot\left|Y_{t}^{(1)}-Y_{t}^{(2)}\right| \lambda(d u) \\
\leq & \frac{1}{4}\left\|Y_{t}^{(1)}-Y_{t}^{(2)}\right\|^{2}+C_{2}\left|Y_{t}^{(1)}-Y_{t}^{(2)}\right|_{L^{4}}^{2}\left|Y_{t}^{(1)}+z_{t}\right|_{L^{4}}^{2}+C_{2}\left|Y_{t}^{(1)}-Y_{t}^{(2)}\right|_{L^{4}}^{2}\left|Y_{t}^{(2)}+z_{t}\right|_{L^{4}}^{2} \\
& +\left[\left|Y_{t}^{(1)}+z_{t}\right|+\left|Y_{t}^{(2)}+z_{t}\right|\right]\left|Y_{t}^{(1)}-Y_{t}^{(2)}\right|^{2}+\lambda(U)\left|Y_{t}^{(1)}-Y_{t}^{(2)}\right|^{2}+\frac{1}{\lambda^{\alpha}}\left|Y_{t}^{(1)}-Y_{t}^{(2)}\right|^{2} \\
\leq & \frac{1}{4}\left\|Y_{t}^{(1)}-Y_{t}^{(2)}\right\|^{2}+C_{2}\left\|Y_{t}^{(1)}-Y_{t}^{(2)}\right\|\left|Y_{t}^{(1)}-Y_{t}^{(2)}\right|\left|Y_{t}^{(1)}+z_{t}\right|_{L^{4}}^{2} \\
& +C_{2}\left\|Y_{t}^{(1)}-Y_{t}^{(2)}\right\|\left|Y_{t}^{(1)}-Y_{t}^{(2)}\right|\left|Y_{t}^{(2)}+z_{t}\right|_{L^{4}}^{2} \\
& +\left[\left|Y_{t}^{(1)}+z_{t}\right|+\left|Y_{t}^{(2)}+z_{t}\right|\right]\left|Y_{t}^{(1)}-Y_{t}^{(2)}\right|^{2}+\lambda(U)\left|Y_{t}^{(1)}-Y_{t}^{(2)}\right|^{2}+\frac{1}{\lambda^{\alpha}}\left|Y_{t}^{(1)}-Y_{t}^{(2)}\right|^{2} \\
\leq & \frac{1}{2}\left\|Y_{t}^{(1)}-Y_{t}^{(2)}\right\|^{2}+C_{3}\left[\left|Y_{t}^{(1)}+z_{t}\right|_{L^{4}}^{4}+\left|Y_{t}^{(2)}+z_{t}\right|_{L^{4}}^{4}\right]\left|Y_{t}^{(1)}-Y_{t}^{(2)}\right|^{2} \\
& {\left[\left|Y_{t}^{(1)}+z_{t}\right|+\left|Y_{t}^{(2)}+z_{t}\right|\right]\left|Y_{t}^{(1)}-Y_{t}^{(2)}\right|^{2}+\lambda(U)\left|Y_{t}^{(1)}-Y_{t}^{(2)}\right|^{2}+\frac{1}{\lambda^{\alpha}}\left|Y_{t}^{(1)}-Y_{t}^{(2)}\right|^{2} . } \\
&
\end{aligned}
$$

Applying Gronwall's inequality, we arrive at the uniqueness.

Theorem 3.2 If hypotheses $\left(\mathrm{H}_{1}\right)-\left(\mathrm{H}_{3}\right)$ hold, then for $\forall x \in \mathscr{D}\left(A^{\alpha}\right)$, there exists a unique solution $X$ of (2.3) such that, for a.s. $\omega \in \Omega$,

$$
X .(\omega)-z .(\omega) \in C\left([0, T], \mathscr{D}\left(A^{\alpha}\right)\right) \cap L^{\frac{4}{1-2 \alpha}}\left(0, T ; \mathscr{D}\left(A^{\frac{1}{4}+\frac{\alpha}{2}}\right)\right) \cap L^{2}\left(0, T ; \mathscr{D}\left(A^{\frac{1}{2}+\alpha}\right)\right) .
$$

The transition semigroup associated with (2.3) is a Feller Markov process.

Proof Theorem 3.1 yields that for $x \in \mathscr{D}\left(A^{\alpha}\right)$, equation (3.2) has a unique solution $X$ satisfying $X .-z, \in C\left([0, T], \mathscr{D}\left(A^{\alpha}\right)\right) \cap L^{\frac{4}{1-2 \alpha}}\left(0, T ; \mathscr{D}\left(A^{\frac{1}{4}+\frac{\alpha}{2}}\right)\right) \cap L^{2}\left(0, T ; \mathscr{D}\left(A^{\frac{1}{2}+\alpha}\right)\right)$. Hence we can define on $\left[0, \sigma_{1}\right]$ that

$$
X_{t}^{(1)}= \begin{cases}X(t), & t<\sigma_{1} \\ X_{\sigma_{1-}}+F\left(X_{\sigma_{1}-}, P_{\sigma_{1}}\right), & t=\sigma_{1}\end{cases}
$$

where $P_{t}$ is a stable Poisson point process on $\mathbf{R}^{+} \times U$ with intensity measure $\lambda(d u) d t$.

Next, on $\left[\sigma_{1}, \sigma_{2}\right)$ define

$$
\begin{aligned}
& \tilde{X}_{0}=X_{\sigma_{1}}^{(1)} I_{\left(\sigma_{1}<\infty\right)}, \quad \tilde{\sigma}_{2}=\left(\sigma_{2}-\sigma_{1}\right) I_{\sigma_{1}<\infty}+\infty I_{\left(\sigma_{1}=\infty\right)}, \\
& \tilde{\mathscr{F}}_{t}=\mathscr{F}_{\sigma_{1}+t}, \quad \tilde{P}(t)=\left(\theta_{\sigma_{1}} P\right)(t) I_{\left(\sigma_{1}<\infty\right)} .
\end{aligned}
$$


$\tilde{P}_{t}$ is still a stable point process with intensity measure $\lambda(d u) d t$. Likewise, equation (3.2) has a unique solution $\tilde{X}$ satisfying $\tilde{X} .-z . \in C\left([0, T], \mathscr{D}\left(A^{\alpha}\right)\right) \cap L^{\frac{4}{1-2 \alpha}}\left(0, T ; \mathscr{D}\left(A^{\frac{1}{4}+\frac{\alpha}{2}}\right)\right) \cap$ $L^{2}\left(0, T ; \mathscr{D}\left(A^{\frac{1}{2}+\alpha}\right)\right)$ corresponding to the initial value $\tilde{X}_{0}$. It is reasonable to define on $\left[0, \sigma_{2}\right]$ that

$$
X_{t}^{(2)}= \begin{cases}X^{(1)}(t), & t \leq \sigma_{1}, \\ \tilde{X}_{t-\sigma_{1}}, & \sigma_{1}<t<\sigma_{2}, \\ \tilde{X}_{\left(\sigma_{2}-\sigma_{1}\right)-}+F\left(\tilde{X}_{\left(\sigma_{2}-\sigma_{1}\right)}, P_{\sigma_{2}}\right), & t=\sigma_{2}\end{cases}
$$

Fixing $T>0$, the Poisson point process $P_{t}$ has only finite jumps on $[0, T]$. Therefore, choosing the first integer $N$ to satisfy $\sigma_{N+1} \geq T$, the method above can be iterated finite times to get $X_{t}^{(N)}$. It is straightforward to show that $X_{t}^{(N)}$ is the unique solution satisfying equation (2.3).

\section{2 $\lambda(\cdot)$ being the $\sigma$-finite measure}

Theorem 3.3 If hypotheses $\left(\mathrm{H}_{1}\right)-\left(\mathrm{H}_{3}\right)$ hold, then for $\forall x \in \mathscr{D}\left(A^{\alpha}\right)$, there exists a unique solution $X$ of (2.3) such that, for a.s. $\omega \in \Omega$,

$$
X .(\omega)-z .(\omega) \in C\left([0, T], \mathscr{D}\left(A^{\alpha}\right)\right) \cap L^{\frac{4}{1-2 \alpha}}\left(0, T ; \mathscr{D}\left(A^{\frac{1}{4}+\frac{\alpha}{2}}\right)\right) \cap L^{2}\left(0, T ; \mathscr{D}\left(A^{\frac{1}{2}+\alpha}\right)\right) .
$$

The transition semigroup associated with (2.3) is a Feller Markov process.

Proof For every $n \geq 1$, consider the equation

$$
\left\{\begin{array}{l}
d Y_{t}^{n}+A Y_{t}^{n} d t+B\left(Y_{t}^{n}+z_{t}\right) d t+R\left(Y_{t}^{n}+z_{t}\right) d t=\int_{U_{n}} F\left(Y_{t-}^{n}+z_{t-}, u\right) \tilde{N}(d t, d u) \\
Y_{0}^{n}=x .
\end{array}\right.
$$

Hereafter, we define $\Xi Z(t):=Z(t)-Z(t-)$. By Itô’s formula in [12], one finds that

$$
\begin{aligned}
\left|Y_{t}^{n}\right|^{2} & \\
=|x|^{2} & +\int_{0}^{t}\left(2 Y_{s-}^{n}, d Y_{s}^{n}\right)+\sum_{s \leq t}\left(\Xi\left(\left|Y_{s}^{n}\right|^{2}\right)-\left(2 Y_{s-}^{n}, \Xi Y_{s}^{n}\right)\right) \\
= & |x|^{2}+\int_{0}^{t}\left(2 Y_{s-}^{n},-\left[A Y_{s}^{n}+B\left(Y_{s}^{n}+z_{s}\right)+R\left(Y_{s}^{n}+z_{s}\right)\right] d s\right) \\
& +\int_{0}^{t} \int_{U_{n}}\left(2 Y_{s-}^{n}, F\left(Y_{s}^{n}+z_{s}, u\right)\right) \tilde{N}(d s, d u) \\
& +\int_{0}^{t} \int_{U_{n}}\left(\left|\left(Y_{s-}^{n}+F\left(Y_{s}^{n}+z_{s}, u\right)\right)\right|^{2}-\left|Y_{s-}^{n}\right|^{2}\right) N(d s, d u) \\
& \quad-\int_{0}^{t} \int_{U_{n}}\left(2 Y_{s-}^{n}, F\left(Y_{s}^{n}+z_{s}, u\right)\right) N(d s, d u) \\
=|x|^{2} & +\int_{0}^{t}\left(2 Y_{s-}^{n},-\left[A Y_{s}^{n}+B\left(Y_{s}^{n}+z_{s}\right)+R\left(Y_{s}^{n}+z_{s}\right)\right] d s\right) \\
& +\int_{0}^{t} \int_{U_{n}}\left|\left(2 Y_{s-}^{n}, F\left(Y_{s-}^{n}+z_{s-}, u\right)\right)\right| \tilde{N}(d s, d u)
\end{aligned}
$$




$$
\begin{aligned}
& +\int_{0}^{t} \int_{U_{n}} \int_{0}^{1}\left|\left(2\left(Y_{s-}^{n}+s^{\prime} F\left(Y_{s-}^{n}+z_{s-}, u\right)\right), F\left(Y_{s-}^{n}+z_{s-}, u\right)\right)\right| d s^{\prime} N(d s, d u) \\
& +\int_{0}^{t} \int_{U_{n}}\left|\left(2 Y_{s-}^{n}, F\left(Y_{s-}^{n}+z_{s-}, u\right)\right)\right| N(d s, d u) .
\end{aligned}
$$

Therefore,

$$
\begin{aligned}
\left|Y_{t}^{n}\right|^{2} & +2 \int_{0}^{t}\left\|Y_{s}^{n}\right\|^{2} d s \\
\leq & |x|^{2}+2 \int_{0}^{t}\left|\left(B\left(Y_{s}^{n}+z_{s}\right), Y_{s}^{n}\right)\right| d s+2 \int_{0}^{t}\left|\left(R\left(Y_{s}^{n}+z_{s}\right), Y_{s}^{n}\right)\right| d s \\
& +6 \int_{0}^{t} \int_{U_{n}}\left|\left(Y_{s-}^{n}, F\left(Y_{s-}^{n}+z_{s-}, u\right)\right)\right| \tilde{N}(d s, d u) \\
& +2 \int_{0}^{t} \int_{U_{n}}\left|F\left(Y_{s-}^{n}+z_{s-}, u\right)\right|^{2} N(d s, d u) \\
& +2 \int_{0}^{t} \int_{U_{n}}\left|\left(Y_{s-}^{n}, F\left(Y_{s-}^{n}+z_{s-}, u\right)\right)\right| \lambda(d u) d s \\
\leq & |x|^{2}+\int_{0}^{t}\left\|Y_{s}^{n}\right\|^{2} d s+C_{1} \int_{0}^{t}\left|Y_{s}^{n}\right|^{2}\left|z_{s}\right|_{L^{4}}^{4} d s+C_{1} \int_{0}^{t}\left|z_{s}\right|_{L^{4}}^{4} d s+M_{t},
\end{aligned}
$$

where

$$
\begin{aligned}
M_{t}= & 6 \int_{0}^{t} \int_{U_{n}}\left|\left(Y_{s-}^{n}, F\left(Y_{s-}^{n}+z_{s-}, u\right)\right)\right| \tilde{N}(d s, d u) \\
& +2 \int_{0}^{t} \int_{U_{n}}\left|F\left(Y_{s-}^{n}+z_{s-}, u\right)\right|^{2} N(d s, d u) \\
& +2 \int_{0}^{t} \int_{U_{n}}\left|\left(Y_{s-}^{n}, F\left(Y_{s-}^{n}+z_{s-}, u\right)\right)\right| \lambda(d u) d s .
\end{aligned}
$$

Let

$$
\tau_{k_{1}}=\inf \left\{t>0 ; \int_{0}^{t}\left|z_{s}\right|_{L^{4}}^{4} d s>k_{1}\right\}, \quad k_{1}>1 .
$$

Lemma 3.1 yields $\tau_{k_{1}} \nearrow \infty$ as $k_{1} \rightarrow \infty$. Gronwall's inequality implies

$$
\begin{aligned}
& \mathbf{E} \sup _{s \leq \tau_{k_{1}} \wedge t}\left|Y_{s}^{n}\right|^{2}+\mathbf{E} \int_{0}^{\tau_{k_{1}} \wedge t}\left\|Y_{s}^{n}\right\|^{2} d s \\
& \leq {\left[|x|^{2}+C_{1} \mathbf{E} \int_{0}^{\tau_{k_{1}} \wedge t}\left|z_{s}\right|_{L^{4}}^{4} d s+\mathbf{E} \sup _{s \leq \tau_{k_{1}} \wedge t}\left|M_{s}\right|\right] e^{C_{1} k_{1}} } \\
& \leq {\left[|x|^{2}+C_{1} \mathbf{E} \int_{0}^{\tau_{k_{1}} \wedge t}\left|z_{s}\right|_{L^{4}}^{4} d s\right] e^{C_{1} k_{1}} } \\
&+C_{2} e^{C_{1} k_{1}} \mathbf{E} \int_{0}^{\tau_{k_{1}} \wedge t} \int_{U_{n}}\left|\left(Y_{s-}^{n}, F\left(Y_{s-}^{n}+z_{s-}, u\right)\right)\right| \tilde{N}(d s, d u) \\
&+C_{2} e^{C_{1} k_{1}} \mathbf{E} \int_{0}^{\tau_{k_{1}} \wedge t} \int_{U_{n}}\left|F\left(Y_{s-}^{n}+z_{s-}, u\right)\right|^{2} \tilde{N}(d s, d u)
\end{aligned}
$$




$$
\begin{aligned}
& +C_{2} e^{C_{1} k_{1}} \mathbf{E} \int_{0}^{\tau_{k_{1}} \wedge t} \int_{U_{n}}\left|\left(Y_{s-}^{n}, F\left(Y_{s-}^{n}+z_{s-}, u\right)\right)\right| \lambda(d u) d s \\
& +C_{2} e^{C_{1} k_{1}} \mathbf{E} \int_{0}^{\tau_{k_{1}} \wedge t} \int_{U_{n}}\left|F\left(Y_{s-}^{n}+z_{s-}, u\right)\right|^{2} \lambda(d u) d s \\
\leq & {\left[|x|^{2}+C_{1} \mathbf{E} \int_{0}^{\tau_{k_{1}} \wedge t}\left|z_{s}\right|_{L^{4}}^{4} d s\right] e^{C_{1} k_{1}} } \\
& +\varepsilon \mathbf{E} \sup _{s \leq \tau_{k_{1}} \wedge t}\left|Y_{s}^{n}\right|^{2}+C_{3}\left(\lambda_{1}, \varepsilon, k_{1}\right) \mathbf{E} \int_{0}^{\tau_{k_{1}} \wedge t} \int_{U_{n}}\left|A^{\alpha} F\left(Y_{s-}^{n}+z_{s-}, u\right)\right|^{2} \lambda(d u) d s \\
\leq & {\left[|x|^{2}+C_{1} \mathbf{E} \int_{0}^{\tau_{k_{1}} \wedge t}\left|z_{s}\right|_{L^{4}}^{4} d s\right] e^{C_{1} k_{1}} } \\
& +\varepsilon \mathbf{E} \sup _{s \leq \tau_{k_{1}} \wedge t}\left|Y_{s}^{n}\right|^{2}+2 C_{3}\left(\lambda_{1}, \varepsilon, k_{1}\right) \mathbf{E} \int_{0}^{\tau_{k_{1}} \wedge t}\left[K\left|Y_{s}^{n}+z_{s}\right|^{2}+C\right] d s \\
\leq & {\left[|x|^{2}+C_{1} \mathbf{E} \int_{0}^{\tau_{k_{1}} \wedge t}\left|z_{s}\right|_{L^{4}}^{4} d s\right] e^{C_{1} k_{1}} } \\
& +\varepsilon \mathbf{E} \sup _{s \leq \tau_{k_{1}} \wedge t}\left|Y_{s}^{n}\right|^{2}+4 C_{3} K \mathbf{E} \int_{0}^{\tau_{k_{1}} \wedge t}\left|Y_{s}^{n}\right|^{2} d s+4 C_{3} K \mathbf{E} \int_{0}^{\tau_{k_{1}} \wedge t}\left|z_{s}\right|^{2} d s+2 C_{3} C t .
\end{aligned}
$$

This yields

$$
\begin{aligned}
(1-\varepsilon) \mathbf{E} & \sup _{s \leq \tau_{k_{1}} \wedge t}\left|Y_{s}^{n}\right|^{2}+\mathbf{E} \int_{0}^{\tau_{k_{1}} \wedge t}\left\|Y_{s}^{n}\right\|^{2} d s \\
\leq & {\left[|x|^{2}+C_{1} \mathbf{E} \int_{0}^{\tau_{k_{1}} \wedge t}\left|z_{s}\right|_{L^{4}}^{4} d s\right] e^{C_{1} k_{1}} } \\
& +4 C_{3} K \mathbf{E} \int_{0}^{\tau_{k_{1}} \wedge t}\left|Y_{s}^{n}\right|^{2} d s+4 C_{3} K \mathbf{E} \int_{0}^{\tau_{k_{1}} \wedge t}\left|z_{s}\right|^{2} d s+2 C_{3} C t \\
\leq & C_{4}\left(x, \varepsilon, \lambda_{1}, k_{1}, K\right)+C_{5}\left(\varepsilon, \lambda_{1}, k_{1}, K\right) t .
\end{aligned}
$$

Applying Itô’s formula to $\left|A^{\alpha} Y_{t}^{n}\right|^{2}$ implies

$$
\begin{aligned}
& \left|A^{\alpha} Y_{t}^{n}\right|^{2} \\
& =\left|A^{\alpha} x\right|^{2}+\int_{0}^{t}\left(2 A^{\alpha} Y_{s-}^{n} \cdot A^{\alpha},-\left[A Y_{s}^{n}+B\left(Y_{s}^{n}+z_{s}\right)+R\left(Y_{s}^{n}+z_{s}\right)\right] d s\right) \\
& \quad+\int_{0}^{t} \int_{U_{n}}\left(2 A^{\alpha} Y_{s-}^{n} \cdot A^{\alpha}, F\left(Y_{s-}^{n}+z_{s-}, u\right)\right) \tilde{N}(d s, d u) \\
& \quad+\int_{0}^{t} \int_{U_{n}}\left(\left|A^{\alpha}\left(Y_{s-}^{n}+F\left(Y_{s-}^{n}+z_{s-}, u\right)\right)\right|^{2}-\left|A^{\alpha} Y_{s-}^{n}\right|^{2}\right) N(d s, d u) \\
& \quad-\int_{0}^{t} \int_{U_{n}}\left(2 A^{\alpha} Y_{s-}^{n} \cdot A^{\alpha}, F\left(Y_{s-}^{n}+z_{s-}, u\right)\right) N(d s, d u) .
\end{aligned}
$$

Accordingly,

$$
\begin{aligned}
& \left|A^{\alpha} Y_{t}^{n}\right|^{2}+2 \int_{0}^{t}\left|A^{\frac{1}{2}+\alpha} Y_{s}^{n}\right|^{2} d s \\
& \quad \leq\left|A^{\alpha} x\right|^{2}+2 \int_{0}^{t}\left|\left(A^{\alpha-\frac{1}{2}} B\left(Y_{s}^{n}+z_{s}\right), A^{\frac{1}{2}+\alpha} Y_{s}^{n}\right)\right| d s+2 \int_{0}^{t}\left|\left(A^{\alpha} R\left(Y_{s}^{n}+z_{s}\right), A^{\alpha} Y_{s}^{n}\right)\right| d s
\end{aligned}
$$




$$
\begin{aligned}
& \quad+6 \int_{0}^{t} \int_{U_{n}}\left|\left(A^{\alpha} Y_{s-}^{n}, A^{\alpha} F\left(Y_{s-}^{n}+z_{s-}, u\right)\right)\right| \tilde{N}(d s, d u) \\
& +2 \int_{0}^{t} \int_{U_{n}}\left|A^{\alpha} F\left(Y_{s-}^{n}+z_{s-}, u\right)\right|^{2} N(d s, d u) \\
& +2 \int_{0}^{t} \int_{U_{n}}\left|\left(A^{\alpha} Y_{s-}^{n}, A^{\alpha} F\left(Y_{s-}^{n}+z_{s-}, u\right)\right)\right| \lambda(d u) d s \\
& \leq\left|A^{\alpha} x\right|^{2}+\int_{0}^{t} C_{1}\left|A^{\frac{1}{4}+\frac{\alpha}{2}}\left(Y_{s}^{n}+z_{s}\right)\right|^{2}\left|A^{\alpha+\frac{1}{2}} Y_{s}^{n}\right| d s+\int_{0}^{t}\left|A^{\alpha}\left(Y_{s}^{n}+z_{s}^{n}\right)\right|\left|A^{\alpha} Y_{s}^{n}\right| d s+\bar{M}_{t} \\
& \leq\left|A^{\alpha} x\right|^{2}+C_{1} \int_{0}^{t}\left[\left|A^{\alpha} Y_{s}^{n}\right|\left|A^{\frac{1}{2}} Y_{s}^{n}\right|\left|A^{\alpha+\frac{1}{2}} Y_{s}^{n}\right|+\left|A^{\frac{1}{4}+\frac{\alpha}{2}} z_{s}\right|^{2}\left|A^{\alpha+\frac{1}{2}} Y_{s}^{n}\right|\right] d s \\
& \quad+\int_{0}^{t}\left[\left|A^{\alpha} Y_{s}^{n}\right|^{2}+\left|A^{\alpha} Y_{s}^{n}\right|\left|A^{\alpha} z_{s}\right|\right] d s+\bar{M}_{t} \\
& \leq\left|A^{\alpha} x\right|^{2}+\int_{0}^{t}\left[\left|A^{\frac{1}{2}+\alpha} Y_{s}^{n}\right|^{2}+C_{2}\left|A^{\frac{1}{2}} Y_{s}^{n}\right|^{2}\left|A^{\alpha} Y_{s}^{n}\right|^{2}+C_{2}\left|A^{\frac{1}{4}+\frac{\alpha}{2}} z_{s}\right|^{4}\right] d s \\
& \quad+\int_{0}^{t}\left[2\left|A^{\alpha} Y_{s}^{n}\right|^{2}+\left|A^{\alpha} z_{s}\right|^{2}\right] d s+\bar{M}_{t} \\
& \leq\left|A^{\alpha} x\right|^{2}+\int_{0}^{t}\left|A^{\frac{1}{2}+\alpha} Y_{s}^{n}\right|^{2} d s+\int_{0}^{t}\left(2+C_{2}\left|A^{\frac{1}{2}} Y_{s}^{n}\right|^{2}\right)\left|A^{\alpha} Y_{s}^{n}\right|^{2} d s \\
& \quad+\int_{0}^{t}\left[C_{2}\left|A^{\frac{1}{4}+\frac{\alpha}{2}} z_{s}\right|^{4}+\left|A^{\alpha} z_{s}\right|^{2}\right] d s+\bar{M}_{t},
\end{aligned}
$$

where

$$
\begin{aligned}
\bar{M}_{t}= & 6 \int_{0}^{t} \int_{U_{n}}\left|\left(A^{\alpha} Y_{s-}^{n}, A^{\alpha} F\left(Y_{s-}^{n}+z_{s-}, u\right)\right)\right| \tilde{N}(d s, d u) \\
& +2 \int_{0}^{t} \int_{U_{n}}\left|A^{\alpha} F\left(Y_{s-}^{n}+z_{s-}, u\right)\right|^{2} N(d s, d u) \\
& +2 \int_{0}^{t} \int_{U_{n}}\left|\left(A^{\alpha} Y_{s-}^{n}, A^{\alpha} F\left(Y_{s-}^{n}+z_{s-}, u\right)\right)\right| \lambda(d u) d s .
\end{aligned}
$$

Fixing $k_{1}$ first, let

$$
\tau_{k_{2}}^{n}=\tau_{k_{2}, k_{1}}^{n}=\inf \left\{t>0 ; \int_{0}^{t \wedge \tau_{k_{1}}}\left\|Y_{s}^{n}\right\|^{2} d s>k_{2}\right\} \wedge \tau_{k_{1}}, \quad k_{2}>1 .
$$

Equation (3.9) yields $\tau_{k_{2}}^{n} \nearrow \tau_{k_{1}}$ as $k_{2} \rightarrow \infty$. By Gronwall's inequality, we have

$$
\begin{aligned}
\mathbf{E} & \sup _{s \leq \tau_{k_{2}}^{n} \wedge t}\left|A^{\alpha} Y_{s}^{n}\right|^{2}+\mathbf{E} \int_{0}^{\tau_{k_{2}}^{n} \wedge t}\left|A^{\frac{1}{2}+\alpha} Y_{s}^{n}\right|^{2} d s \\
& \leq\left[\left|A^{\alpha} x\right|^{2}+\mathbf{E} \int_{0}^{\tau_{k_{2}}^{n} \wedge t}\left[C_{2}\left|A^{\frac{1}{4}+\frac{\alpha}{2}} z_{s}\right|^{4}+\left|A^{\alpha} z_{s}\right|^{2}\right] d s+\mathbf{E} \sup _{s \leq \tau_{k_{2}}^{n} \wedge t}\left|\bar{M}_{s}\right|\right] e^{2+C_{2} k_{2}} \\
& \leq\left[\left|A^{\alpha} x\right|^{2}+\mathbf{E} \int_{0}^{\tau_{k_{2}}^{n} \wedge t}\left[C_{2}\left|A^{\frac{1}{4}+\frac{\alpha}{2}} z_{s}\right|^{4}+\left|A^{\alpha} z_{s}\right|^{2}\right] d s\right] e^{2+C_{2} k_{2}}+e^{2+C_{2} k_{2}} \mathbf{E} \sup _{s \leq \tau_{k_{2}}^{n} \wedge t}\left|\bar{M}_{s}\right| \\
& \leq\left[\left|A^{\alpha} x\right|^{2}+\mathbf{E} \int_{0}^{\tau_{k_{2}}^{n} \wedge t}\left[C_{2}\left|A^{\frac{1}{4}+\frac{\alpha}{2}} z_{s}\right|^{4}+\left|A^{\alpha} z_{s}\right|^{2}\right] d s\right] e^{2+C_{2} k_{2}}
\end{aligned}
$$




$$
\begin{aligned}
& +C_{3} e^{2+C_{2} k_{2}} \mathbf{E} \int_{0}^{\tau_{k_{2}}^{n} \wedge t} \int_{U_{n}}\left|\left(A^{\alpha} Y_{s-}^{n}, A^{\alpha} F\left(Y_{s-}^{n}+z_{s-}, u\right)\right)\right| \tilde{N}(d s, d u) \\
& +C_{3} e^{2+C_{2} k_{2}} \mathbf{E} \int_{0}^{\tau_{k_{2}}^{n} \wedge t} \int_{U_{n}}\left|A^{\alpha} F\left(Y_{s-}^{n}+z_{s-}, u\right)\right|^{2} \tilde{N}(d s, d u) \\
& +C_{3} e^{2+C_{2} k_{2}} \mathbf{E} \int_{0}^{\tau_{k_{2}}^{n} \wedge t} \int_{U_{n}}\left|\left(A^{\alpha} Y_{s-}^{n}, A^{\alpha} F\left(Y_{s-}^{n}+z_{s-}, u\right)\right)\right| \lambda(d u) d s \\
& +C_{3} e^{2+C_{2} k_{2}} \mathbf{E} \int_{0}^{\tau_{k_{2}}^{n} \wedge t} \int_{U_{n}}\left|A^{\alpha} F\left(Y_{s-}^{n}+z_{s-}, u\right)\right|^{2} \lambda(d u) d s \\
\leq & {\left[\left|A^{\alpha} x\right|^{2}+\mathbf{E} \int_{0}^{\tau_{k_{2}}^{n} \wedge t}\left[C_{2}\left|A^{\frac{1}{4}+\frac{\alpha}{2}} z_{s}\right|^{4}+\left|A^{\alpha} z_{s}\right|^{2}\right] d s\right] e^{2+C_{2} k_{2}} } \\
& +\varepsilon \mathbf{E} \sup _{s \leq \tau_{k_{2}}^{n} \wedge t}\left|A^{\alpha} Y_{s}^{n}\right|^{2}+C_{4}(\varepsilon, k) \mathbf{E} \int_{0}^{\tau_{k_{2}}^{n} \wedge t} \int_{U_{n}}\left|A^{\alpha} F\left(Y_{s-}^{n}+z_{s-}, u\right)\right|^{2} \lambda(d u) d s \\
\leq & {\left[\left|A^{\alpha} x\right|^{2}+\mathbf{E} \int_{0}^{\tau_{k_{2}}^{n} \wedge t}\left[C_{2}\left|A^{\frac{1}{4}+\frac{\alpha}{2}} z_{s}\right|^{4}+\left|A^{\alpha} z_{s}\right|^{2}\right] d s\right] e^{2+C_{2} k_{2}} } \\
& +\varepsilon \mathbf{E} \sup _{s \leq \tau_{k_{2}}^{n} \wedge t}\left|A^{\alpha} Y_{s}^{n}\right|^{2}+2 C_{4}(\varepsilon, k) \mathbf{E} \int_{0}^{\tau_{k_{2}}^{n} \wedge t}\left[K\left|Y_{s}^{n}+z_{s}\right|^{2}+C\right] d s \\
\leq & {\left[\left|A^{\alpha} x\right|^{2}+\mathbf{E} \int_{0}^{\tau_{k_{2}}^{n} \wedge t}\left[C_{2}\left|A^{\frac{1}{4}+\frac{\alpha}{2}} z_{s}\right|^{4}+\left|A^{\alpha} z_{s}\right|^{2}\right] d s\right] e^{2+C_{2} k_{2}} } \\
& +\varepsilon \mathbf{E} \sup _{s \leq \tau_{k_{2}}^{n} \wedge t}\left|A^{\alpha} Y_{s}^{n}\right|^{2}+\frac{4 C_{4} K}{\lambda_{1}} \mathbf{E} \int_{0}^{\tau_{k_{2}}^{n} \wedge t}\left\|Y_{s}^{n}\right\|^{2} d s \\
& +4 C_{4} K \mathbf{E} \int_{0}^{\tau_{k_{2}}^{n} \wedge t}\left|z_{s}\right|^{2} d s+2 C_{4} C t . \\
&
\end{aligned}
$$

This yields

$$
\begin{aligned}
(1-\varepsilon) \mathbf{E} & \sup _{s \leq \tau_{k_{2}}^{n} \wedge t}\left|A^{\alpha} Y_{s}^{n}\right|^{2}+\mathbf{E} \int_{0}^{\tau_{k_{2}}^{n} \wedge t}\left|A^{\frac{1}{2}+\alpha} Y_{s}^{n}\right|^{2} d s \\
\leq & {\left[\left|A^{\alpha} x\right|^{2}+\mathbf{E} \int_{0}^{\tau_{k_{2}}^{n} \wedge t}\left[C_{2}\left|A^{\frac{1}{4}+\frac{\alpha}{2}} z_{s}\right|^{4}+\left|A^{\alpha} z_{s}\right|^{2}\right] d s\right] e^{2+C_{2} k_{2}} } \\
& +\frac{4 C_{4} K k_{2}}{\lambda_{1}}+4 C_{4} K \mathbf{E} \int_{0}^{\tau_{k_{2}}^{n} \wedge t}\left|z_{s}\right|^{2} d s+2 C_{4} C t \\
\leq & C_{5}\left(x, \varepsilon, \alpha, \lambda_{1}, k_{2}, K\right)+C_{6}\left(\varepsilon, \alpha, \lambda_{1}, k_{2}, K\right) t .
\end{aligned}
$$

For every $n, m \geq 1, Y_{t}^{n}-Y_{t}^{m}$ satisfies the equation

$$
\left\{\begin{array}{l}
d\left(Y_{t}^{n}-Y_{t}^{m}\right)+A\left(Y_{t}^{n}-Y_{t}^{m}\right) d t+B\left(Y_{t}^{n}+z_{t}\right) d t \\
\quad-B\left(Y_{t}^{m}+z_{t}\right) d t+R\left(Y_{t}^{n}-Y_{t}^{m}\right) d t \\
=\int_{U_{n} \backslash U_{m}} F\left(Y_{t-}^{n}+z_{t-}, u\right) \tilde{N}(d t, d u) \\
\quad+\int_{U_{m}}\left[F\left(Y_{t-}^{n}+z_{t-}, u\right)-F\left(Y_{t-}^{m}+z_{t-}, u\right)\right] \tilde{N}(d t, d u), \\
Y_{0}^{n}-Y_{0}^{m}=0 .
\end{array}\right.
$$


Recall that $\Xi Z(t):=Z(t)-Z(t-)$. Applying Itô's formula to $\left|A^{\alpha}\left(Y_{t}^{n}-Y_{t}^{m}\right)\right|^{2}$, we have

$$
\begin{aligned}
\mid A^{\alpha}( & \left.Y_{t}^{n}-Y_{t}^{m}\right)\left.\right|^{2} \\
= & \int_{0}^{t}\left(2 A^{\alpha}\left(Y_{s-}^{n}-Y_{s-}^{m}\right) \cdot A^{\alpha}, d\left(Y_{s}^{n}-Y_{s}^{m}\right)\right) \\
& +\sum_{s \leq t}\left(\Xi\left(\left|A^{\alpha}\left(Y_{s}^{n}-Y_{s}^{m}\right)\right|^{2}\right)-\left(2 A^{\alpha}\left(Y_{s-}^{n}-Y_{s-}^{m}\right) \cdot A^{\alpha}, \Xi\left(Y_{s}^{n}-Y_{s}^{m}\right)\right)\right) \\
= & \int_{0}^{t}\left(2 A^{\alpha}\left(Y_{s-}^{n}-Y_{s-}^{m}\right) \cdot A^{\alpha},-\left[A\left(Y_{s}^{n}-Y_{s}^{m}\right)+B\left(Y_{s}^{n}+z_{s}\right)\right.\right. \\
& \left.\left.-B\left(Y_{s}^{m}+z_{s}\right)+R\left(Y_{s}^{n}-Y_{s}^{m}\right)\right] d s\right) \\
& +\int_{0}^{t} \int_{U_{n}}\left(2 A^{\alpha}\left(Y_{s-}^{n}-Y_{s-}^{m}\right) \cdot A^{\alpha}, F\left(Y_{s-}^{n}+z_{s-}, u\right)\right) \tilde{N}(d s, d u) \\
& -\int_{0}^{t} \int_{U_{m}}\left(2 A^{\alpha}\left(Y_{s-}^{n}-Y_{s-}^{m}\right) \cdot A^{\alpha}, F\left(Y_{s-}^{m}+z_{s-}, u\right)\right) \tilde{N}(d s, d u) \\
& +\int_{0}^{t} \int_{U_{n} \backslash U_{m}}\left(\left|A^{\alpha}\left(\left(Y_{s-}^{n}-Y_{s-}^{m}\right)+F\left(Y_{s-}^{n}+z_{s-}, u\right)\right)\right|^{2}-\left|A^{\alpha}\left(Y_{s-}^{n}-Y_{s-}^{m}\right)\right|^{2}\right) N(d s, d u) \\
& +\int_{0}^{t} \int_{U_{m}}\left(\left|A^{\alpha}\left(\left(Y_{s-}^{n}-Y_{s-}^{m}\right)+F\left(Y_{s-}^{n}+z_{s-}, u\right)-F\left(Y_{s-}^{m}+z_{s-}, u\right)\right)\right|^{2}\right. \\
& \left.-\left|A^{\alpha}\left(Y_{s-}^{n}-Y_{s-}^{m}\right)\right|^{2}\right) N(d s, d u) \\
& -\int_{0}^{t} \int_{U_{n}}\left(2 A^{\alpha}\left(Y_{s-}^{n}-Y_{s-}^{m}\right) \cdot A^{\alpha}, F\left(Y_{s-}^{n}+z_{s-}, u\right)\right) N(d s, d u) \\
& +\int_{0}^{t} \int_{U_{m}}\left(2 A^{\alpha}\left(Y_{s-}^{n}-Y_{s-}^{m}\right) \cdot A^{\alpha}, F\left(Y_{s-}^{m}+z_{s-}, u\right)\right) N(d s, d u)
\end{aligned}
$$

Therefore

$$
\begin{aligned}
& \left|A^{\alpha}\left(Y_{t}^{n}-Y_{t}^{m}\right)\right|^{2}+2 \int_{0}^{t}\left|A^{\frac{1}{2}+\alpha}\left(Y_{s}^{n}-Y_{s}^{m}\right)\right|^{2} d s \\
& \leq 2 \int_{0}^{t}\left|\left(B\left(Y_{s}^{n}+z_{s}\right), A^{2 \alpha}\left(Y_{s}^{n}-Y_{s}^{m}\right)\right)\right| d s+2 \int_{0}^{t}\left|\left(B\left(Y_{s}^{m}+z_{s}\right), A^{2 \alpha}\left(Y_{s}^{n}-Y_{s}^{m}\right)\right)\right| d s \\
& \quad+2 \int_{0}^{t}\left|\left(A^{\alpha} R\left(Y_{s}^{n}-Y_{s}^{m}\right), A^{\alpha}\left(Y_{s}^{n}-Y_{s}^{m}\right)\right)\right| d s \\
& \quad+6 \int_{0}^{t} \int_{U_{n} \backslash U_{m}}\left|\left(A^{\alpha}\left(Y_{s-}^{n}-Y_{s-}^{m}\right), A^{\alpha} F\left(Y_{s-}^{n}+z_{s-}, u\right)\right)\right| \tilde{N}(d s, d u) \\
& \quad+6 \int_{0}^{t} \int_{U_{m}}\left|\left(A^{\alpha}\left(Y_{s-}^{n}-Y_{s-}^{m}\right), A^{\alpha}\left(F\left(Y_{s-}^{n}+z_{s-}, u\right)-F\left(Y_{s-}^{m}+z_{s-}, u\right)\right)\right)\right| \tilde{N}(d s, d u) \\
& \quad+2 \int_{0}^{t} \int_{U_{n} \backslash U_{m}}\left|A^{\alpha} F\left(Y_{s-}^{n}+z_{s-}, u\right)\right|^{2} N(d s, d u) \\
& \quad+2 \int_{0}^{t} \int_{U_{m}}\left|A^{\alpha}\left(F\left(Y_{s-}^{n}+z_{s-}, u\right)-F\left(Y_{s-}^{m}+z_{s-}, u\right)\right)\right|^{2} N(d s, d u) \\
& \quad+2 \int_{0}^{t} \int_{U_{n} \backslash U_{m}}\left|\left(A^{\alpha}\left(Y_{s-}^{n}-Y_{s-}^{m}\right), A^{\alpha} F\left(Y_{s-}^{n}+z_{s-}, u\right)\right)\right| \lambda(d u) d s
\end{aligned}
$$




$$
\begin{aligned}
& +2 \int_{0}^{t} \int_{U_{m}}\left|\left(A^{\alpha}\left(Y_{s-}^{n}-Y_{s-}^{m}\right), A^{\alpha}\left(F\left(Y_{s-}^{n}+z_{s-}, u\right)-F\left(Y_{s-}^{m}+z_{s-}, u\right)\right)\right)\right| \lambda(d u) d s \\
\leq & C_{1} \int_{0}^{t}\left|A^{\alpha+\frac{1}{2}}\left(Y_{s}^{n}-Y_{s}^{m}\right)\right|^{\frac{3}{2}-\alpha}\left|A^{\alpha}\left(Y_{s}^{n}-Y_{s}^{m}\right)\right|^{\frac{1}{2}+\alpha} \\
& \times\left[\left|A^{\frac{1}{4}+\frac{\alpha}{2}}\left(Y_{s}^{n}+z_{s}\right)\right|+\left|A^{\frac{1}{4}+\frac{\alpha}{2}}\left(Y_{s}^{m}+z_{s}\right)\right|\right] d s+2 \int_{0}^{t}\left|A^{\alpha}\left(Y_{s}^{n}-Y_{s}^{m}\right)\right|^{2} d s+g_{m, n}(t) \\
\leq & \int_{0}^{t}\left|A^{\alpha+\frac{1}{2}}\left(Y_{s}^{n}-Y_{s}^{m}\right)\right|^{2} d s \\
& +C_{2} \int_{0}^{t}\left|A^{\alpha}\left(Y_{s}^{n}-Y_{s}^{m}\right)\right|^{2}\left[\left|A^{\frac{1}{4}+\frac{\alpha}{2}}\left(Y_{s}^{n}+z_{s}\right)\right|+\left|A^{\frac{1}{4}+\frac{\alpha}{2}}\left(Y_{s}^{m}+z_{s}\right)\right|\right]^{\frac{1+2 \alpha}{4}} d s \\
& +2 \int_{0}^{t}\left|A^{\alpha}\left(Y_{s}^{n}-Y_{s}^{m}\right)\right|^{2} d s+g_{m, n}(t),
\end{aligned}
$$

where

$$
\begin{aligned}
g_{m, n}(t)= & 6 \int_{0}^{t} \int_{U_{n} \backslash U_{m}}\left|\left(A^{\alpha}\left(Y_{s-}^{n}-Y_{s-}^{m}\right), A^{\alpha} F\left(Y_{s-}^{n}+z_{s-}, u\right)\right)\right| \tilde{N}(d s, d u) \\
& +6 \int_{0}^{t} \int_{U_{m}}\left|\left(A^{\alpha}\left(Y_{s-}^{n}-Y_{s-}^{m}\right), A^{\alpha}\left(F\left(Y_{s-}^{n}+z_{s-}, u\right)-F\left(Y_{s-}^{m}+z_{s-}, u\right)\right)\right)\right| \tilde{N}(d s, d u) \\
& +2 \int_{0}^{t} \int_{U_{n} \backslash U_{m}}\left|A^{\alpha} F\left(Y_{s-}^{n}+z_{s-}, u\right)\right|^{2} N(d s, d u) \\
& +2 \int_{0}^{t} \int_{U_{m}}\left|A^{\alpha}\left(F\left(Y_{s-}^{n}+z_{s-}, u\right)-F\left(Y_{s-}^{m}+z_{s-}, u\right)\right)\right|^{2} N(d s, d u) \\
& +2 \int_{0}^{t} \int_{U_{n} \backslash U_{m}}\left|\left(A^{\alpha}\left(Y_{s-}^{n}-Y_{s-}^{m}\right), A^{\alpha} F\left(Y_{s-}^{n}+z_{s-}, u\right)\right)\right| \lambda(d u) d s \\
& +2 \int_{0}^{t} \int_{U_{m}}\left|\left(A^{\alpha}\left(Y_{s-}^{n}-Y_{s-}^{m}\right), A^{\alpha}\left(F\left(Y_{s-}^{n}+z_{s-}, u\right)-F\left(Y_{s-}^{m}+z_{s-}, u\right)\right)\right)\right| \lambda(d u) d s .
\end{aligned}
$$

Now fixing $k_{1}, k_{2}$ consecutively, let

$$
\begin{aligned}
\tau_{k_{3}}^{m, n}= & \tau_{k_{3}, k_{2}, k_{1}}^{m, n} \\
= & \inf \left\{t>0 ; \int_{0}^{t \wedge \tau_{k_{2}}^{n} \wedge \tau_{k_{2}}^{m}}\left[\left|A^{\frac{1}{4}+\frac{\alpha}{2}}\left(Y_{s}^{n}+z_{s}\right)\right|+\left|A^{\frac{1}{4}+\frac{\alpha}{2}}\left(Y_{s}^{m}+z_{s}\right)\right|\right]^{\frac{1+2 \alpha}{4}} d s>k_{3}\right\} \\
& \wedge \tau_{k_{2}}^{n} \wedge \tau_{k_{2}}^{m}, \quad k_{3}>1 .
\end{aligned}
$$

Equations (3.5), (3.10), and Lemma 3.1 imply that $\tau_{k_{3}}^{m, n} \nearrow \tau_{k_{2}}^{n} \wedge \tau_{k_{2}}^{m}$ as $k_{3} \rightarrow \infty$. By Gronwall's inequality, one gets

$$
\begin{aligned}
& \mathbf{E} \sup _{s \leq \tau_{k_{3}}^{m, n} \wedge t}\left|A^{\alpha}\left(Y_{s}^{n}-Y_{s}^{m}\right)\right|^{2}+\mathbf{E} \int_{0}^{\tau_{k_{3}}^{m, n} \wedge t}\left|A^{\frac{1}{2}+\alpha}\left(Y_{s}^{n}-Y_{s}^{m}\right)\right|^{2} d s \\
& \quad \leq e^{2+C_{2} k_{3}} \mathbf{E} \sup _{s \leq \tau_{k_{3}}^{m, n} \wedge t}\left|g_{m, n}(s)\right| \\
& \quad \leq C_{3} e^{2+C_{2} k_{3}} \mathbf{E} \int_{0}^{\tau_{k_{3}}^{m, n} \wedge t} \int_{U_{n} \backslash U_{m}}\left|\left(A^{\alpha}\left(Y_{s-}^{n}-Y_{s-}^{m}\right), A^{\alpha} F\left(Y_{s-}^{n}+z_{s-}, u\right)\right)\right| \tilde{N}(d s, d u)
\end{aligned}
$$




$$
\begin{aligned}
& +C_{3} e^{2+C_{2} k_{3}} \mathbf{E} \int_{0}^{\tau_{k_{3}}^{m, n} \wedge t} \int_{U_{n} \backslash U_{m}}\left|A^{\alpha} F\left(Y_{s-}^{n}+z_{s-}, u\right)\right|^{2} \tilde{N}(d s, d u) \\
& +C_{3} e^{2+C_{2} k_{3}} \mathbf{E} \int_{0}^{\tau_{k_{3}}^{m, n} \wedge t} \int_{U_{n} \backslash U_{m}}\left|\left(A^{\alpha}\left(Y_{s-}^{n}-Y_{s-}^{m}\right), A^{\alpha} F\left(Y_{s-}^{n}+z_{s-}, u\right)\right)\right| \lambda(d u) d s \\
& +C_{3} e^{2+C_{2} k_{3}} \mathbf{E} \int_{0}^{\tau_{k_{3}}^{m, n} \wedge t} \int_{U_{n} \backslash U_{m}}\left|A^{\alpha} F\left(Y_{s-}^{n}+z_{s-}, u\right)\right|^{2} \lambda(d u) d s \\
& +C_{3} e^{2+C_{2} k_{3}} \mathbf{E} \int_{0}^{\tau_{k_{3}}^{m, n} \wedge t} \int_{U_{m}} \mid\left(A^{\alpha}\left(Y_{s-}^{n}-Y_{s-}^{m}\right), A^{\alpha} F\left(Y_{s-}^{n}+z_{s-}, u\right)\right. \\
& \left.-A^{\alpha} F\left(Y_{s-}^{n}+z_{s-}, u\right)\right) \mid \tilde{N}(d s, d u) \\
& +C_{3} e^{2+C_{2} k_{3}} \mathbf{E} \int_{0}^{\tau_{k_{3}}^{m, n} \wedge t} \int_{U_{m}}\left|A^{\alpha} F\left(Y_{s-}^{n}+z_{s-}, u\right)-A^{\alpha} F\left(Y_{s-}^{n}+z_{s-}, u\right)\right|^{2} \tilde{N}(d s, d u) \\
& +C_{3} e^{2+C_{2} k_{3}} \mathbf{E} \int_{0}^{\tau_{k_{3}}^{m, n} \wedge t} \int_{U_{m}} \mid\left(A^{\alpha}\left(Y_{s-}^{n}-Y_{s-}^{m}\right), A^{\alpha} F\left(Y_{s-}^{n}+z_{s-}, u\right)\right. \\
& \left.-A^{\alpha} F\left(Y_{s-}^{n}+z_{s-}, u\right)\right) \mid \lambda(d u) d s \\
& +C_{3} e^{2+C_{2} k_{3}} \mathbf{E} \int_{0}^{\tau_{k_{3}}^{m, n} \wedge t} \int_{U_{m}}\left|A^{\alpha} F\left(Y_{s-}^{n}+z_{s-}, u\right)-A^{\alpha} F\left(Y_{s-}^{n}+z_{s-}, u\right)\right|^{2} \lambda(d u) d s \\
& \leq \varepsilon \mathbf{E} \sup _{s \leq \tau_{k_{3}}^{m, n} \wedge t}\left|A^{\alpha}\left(Y_{s}^{n}-Y_{s}^{m}\right)\right|^{2}+C_{4}(\varepsilon, k) \mathbf{E} \int_{0}^{\tau_{k_{3}}^{m, n} \wedge t} \int_{U_{n} \backslash U_{m}}\left|A^{\alpha} F\left(Y_{s}^{n}+z_{s}, u\right)\right|^{2} \lambda(d u) d s .
\end{aligned}
$$

Noting hypothesis $\left(\mathrm{H}_{4}\right)$, we have

$$
\begin{aligned}
& (1-\varepsilon) \mathbf{E} \sup _{s \leq \tau_{k_{3}}^{m, n} \wedge t}\left|A^{\alpha}\left(Y_{s}^{n}-Y_{s}^{m}\right)\right|^{2}+\mathbf{E} \int_{0}^{\tau_{k_{3}}^{m, n} \wedge t}\left|A^{\frac{1}{2}+\alpha}\left(Y_{s}^{n}-Y_{s}^{m}\right)\right|^{2} d s \\
& \leq C_{4} t \sup _{x \in \mathbf{H}} \int_{U_{m}^{c}}\left|A^{\alpha} F(x, u)\right|^{2} \lambda(d u) \rightarrow 0,
\end{aligned}
$$

as $m \rightarrow \infty$.

Since

$$
\begin{aligned}
& \mathbf{E} \sup _{s \leq \tau_{k_{1}} \wedge t}\left|A^{\alpha}\left(Y_{s}^{n}-Y_{s}^{m}\right)\right| \\
& \quad \leq\left(\mathbf{E} \sup _{s \leq \tau_{k_{3}}^{m, n} \wedge t}\left|A^{\alpha}\left(Y_{s}^{n}-Y_{s}^{m}\right)\right|^{2}\right)^{\frac{1}{2}}+\left(\mathbf{E} \sup _{s \leq \tau_{k_{1}} \wedge t}\left|A^{\alpha}\left(Y_{s}^{n}-Y_{s}^{m}\right)\right|^{2}\right)^{\frac{1}{2}}\left[\mathbf{P}\left(t>\tau_{k_{3}}^{m, n}\right)\right]^{\frac{1}{2}}
\end{aligned}
$$

and

$$
\begin{aligned}
\mathbf{E} \int_{0}^{\tau_{k_{1}} \wedge t}\left|A^{\frac{1}{2}+\alpha}\left(Y_{s}^{n}-Y_{s}^{m}\right)\right| d s \\
\leq\left(\mathbf{E} \int_{0}^{\tau_{k_{3}}^{m, n} \wedge t}\left|A^{\frac{1}{2}+\alpha}\left(Y_{s}^{n}-Y_{s}^{m}\right)\right|^{2} d s\right)^{\frac{1}{2}} \\
\quad+\left(\mathbf{E} \int_{0}^{\tau_{k_{1}} \wedge t}\left|A^{\frac{1}{2}+\alpha}\left(Y_{s}^{n}-Y_{s}^{m}\right)\right|^{2} d s\right)^{\frac{1}{2}}\left[\mathbf{P}\left(t>\tau_{k_{3}}^{m, n}\right)\right]^{\frac{1}{2}},
\end{aligned}
$$


we conclude that, for any fixed $t>0$ and $k_{1} \geq 1$,

$$
\lim _{m \rightarrow \infty, n \geq m}\left[\mathbf{E} \sup _{s \leq \tau_{k_{1}} \wedge t}\left|A^{\alpha}\left(Y_{s}^{n}-Y_{s}^{m}\right)\right|+\mathbf{E} \int_{0}^{\tau_{k_{1}} \wedge t}\left|A^{\frac{1}{2}+\alpha}\left(Y_{s}^{n}-Y_{s}^{m}\right)\right| d s\right]=0 .
$$

So $\left\{Y_{\tau_{k_{1}} \wedge s}^{n}\right\}$ is a Cauchy sequence in the space $\mathscr{D}_{T}$, which is the space of all $\mathscr{D}\left(A^{\alpha}\right)$-valued adapted càdlàg processes $Y_{t}$ with $\mathbf{E}\left(\sup _{t \leq T}\left|A^{\alpha} Y_{t}\right|+\int_{0}^{T}\left|A^{\frac{1}{2}+\alpha} Y_{s}\right| d s\right)<\infty$ for any positive number $T$. Consequently, there exists a process $\bar{Y}^{k_{1}} \in \mathscr{D}_{T}$ such that

$$
\lim _{n \rightarrow \infty}\left[\mathbf{E} \sup _{s \leq \tau_{k_{1}} \wedge T}\left|A^{\alpha}\left(Y_{s}^{n}-\bar{Y}_{s}^{k_{1}}\right)\right|+\mathbf{E} \int_{0}^{\tau_{k_{1}} \wedge t}\left|A^{\frac{1}{2}+\alpha}\left(Y_{s}^{n}-\bar{Y}_{s}^{k_{1}}\right)\right| d s\right]=0 .
$$

It is straightforward to examine that $\bar{Y}^{k_{1}}$ is a weak solution of (2.3) on [0, $\left.\tau_{k_{1}}\right]$ (cf. [6]). Set

$$
Y=\sum_{k_{1}=1}^{\infty} \bar{Y}^{k_{1}} I_{\left\{\left[\tau_{k_{1}-1}, \tau_{k_{1}}\right)\right\}}
$$

where $\tau_{0}=0$. Since $\tau_{k_{1}} \rightarrow \infty(k \rightarrow \infty), Y$ is a weak solution of (2.3).

\section{Invariant measure}

This section aims to show that, although the fragile solution is in a somewhat weaker sense than the classical weak solution, we can utilize it to prove the existence of an invariant measure following classical routes, say by making use of the Krylov-Bogoliubov averaging procedure with energy (compactness) estimates.

Theorem 4.1 Assume that hypotheses $\left(\mathrm{H}_{1}\right)-\left(\mathrm{H}_{4}\right)$ hold, then there exists an invariant measure $\mu$ for the transition semigroup $P_{t}$ associated with (2.3).

Proof Let $X_{t}=X\left(t, t_{0}\right)$ be the solution of the following equation:

$$
\begin{aligned}
X_{t}= & X_{t_{0}}-\int_{t_{0}}^{t} A X_{s} d s-\int_{t_{0}}^{t} B\left(X_{s}\right) d s-\int_{t_{0}}^{t} R\left(X_{s}\right) d s+\int_{t_{0}}^{t} Q d W_{s} \\
& +\int_{t_{0}}^{t} \int_{U} F\left(X_{s}, u\right) \tilde{N}(d s, d u) .
\end{aligned}
$$

Consider the Ornstein-Uhlenbeck process $z_{t}^{\gamma}$ satisfying $d z_{t}^{\gamma}=(-A-\gamma) z_{t}^{\gamma} d t+Q d W_{t}$. Make Ornstein-Uhlenbeck transformation of (4.1) by letting $X\left(t, t_{0}\right)=Z^{\gamma}\left(t, t_{0}\right)+z_{t}^{\gamma}$, then $Z_{t}^{\gamma}=Z^{\gamma}\left(t, t_{0}\right)$ satisfies

$$
\left\{\begin{aligned}
d Z_{t}^{\gamma}= & -A Z_{t}^{\gamma} d t-B\left(Z_{t}^{\gamma}+z_{t}^{\gamma}\right) d t-R\left(Z_{t}^{\gamma}+z_{t}^{\gamma}\right) d t \\
& +\int_{U} F\left(Z_{t}^{\gamma}+z_{t}^{\gamma}, u\right) \tilde{N}(d t, d u) \\
Z_{t_{0}}^{\gamma}= & -z_{t_{0}}^{\gamma} .
\end{aligned}\right.
$$


Recall that $\Xi Z(t):=Z(t)-Z(t-)$. Applying Itô's formula to $\left|A^{\alpha} Z_{t}^{\gamma}(t)\right|^{2}$ yields

$$
\begin{aligned}
\left|A^{\alpha} Z_{t}^{\gamma}\right|^{2}= & \left|A^{\alpha} Z_{t_{0}}^{\gamma}\right|^{2}+\int_{t_{0}}^{t}\left\langle 2 A^{\alpha} Z_{s-}^{\gamma} \cdot A^{\alpha}, d Z_{s}^{\gamma}\right\rangle \\
& +\sum_{s \leq t}\left(\Xi\left(\left|A^{\alpha} Z_{s}^{\gamma}\right|^{2}\right)-\left\langle 2 A^{\alpha} Z_{s-}^{\gamma} \cdot A^{\alpha}, \Xi Z_{s}^{\gamma}\right\rangle\right) \\
= & \left|A^{\alpha} Z_{t_{0}}^{\gamma}\right|^{2}+\int_{t_{0}}^{t}\left(2 A^{\alpha} Z_{s-}^{\gamma} \cdot A^{\alpha},-\left[A Z_{s}^{\gamma}+B\left(Z_{s}^{\gamma}+z_{s}^{\gamma}\right)+R\left(Z_{s}^{\gamma}+z_{s}^{\gamma}\right)\right] d s\right) \\
& +\int_{t_{0}}^{t} \int_{U}\left(2 A^{\alpha} Z_{s-}^{\gamma} \cdot A^{\alpha}, F\left(Z_{s-}^{\gamma}+z_{s-}^{\gamma}, u\right)\right) \tilde{N}(d s, d u) \\
& +\int_{t_{0}}^{t} \int_{U}\left(\left|A^{\alpha}\left(Z_{s-}^{\gamma}+F\left(Z_{s-}^{\gamma}+z_{s-}^{\gamma}, u\right)\right)\right|^{2}-\left|A^{\alpha} Z_{s-}^{\gamma}\right|^{2}\right) N(d s, d u) \\
& -\int_{t_{0}}^{t} \int_{U}\left(2 A^{\alpha} Z_{s-}^{\gamma} \cdot A^{\alpha}, F\left(Z_{s-}^{\gamma}+z_{s-}^{\gamma}, u\right)\right) N(d s, d u) \\
= & \left|A^{\alpha} Z_{t_{0}}^{\gamma}\right|^{2}+\int_{t_{0}}^{t}\left(2 A^{\alpha} Z_{s-}^{\gamma} \cdot A^{\alpha},-\left[A Z_{s}^{\gamma}+B\left(Z_{s}^{\gamma}+z_{s}^{\gamma}\right)+R\left(Z_{s}^{\gamma}+z_{s}^{\gamma}\right)\right] d s\right)+\tilde{M}_{t},
\end{aligned}
$$

where

$$
\begin{aligned}
\tilde{M}_{t}= & \int_{t_{0}}^{t} \int_{U}\left(2 A^{\alpha} Z_{s-}^{\gamma} \cdot A^{\alpha}, F\left(Z_{s-}^{\gamma}+z_{s-}^{\gamma}, u\right)\right) \tilde{N}(d s, d u) \\
& +\int_{t_{0}}^{t} \int_{U}\left(\left|A^{\alpha}\left(Z_{s-}^{\gamma}+F\left(Z_{s-}^{\gamma}+z_{s-}^{\gamma}, u\right)\right)\right|^{2}-\left|A^{\alpha} Z_{s-}^{\gamma}\right|^{2}\right) N(d s, d u) \\
& -\int_{t_{0}}^{t} \int_{U}\left(2 A^{\alpha} Z_{s-}^{\gamma} \cdot A^{\alpha}, F\left(Z_{s-}^{\gamma}+z_{s-}^{\gamma}, u\right)\right) N(d s, d u) .
\end{aligned}
$$

In the following proof, we will utilize the interpolation inequality

$$
\left|\left(B(u, v), A^{2 \alpha} w\right)\right| \leq C_{0}\left|A^{\frac{1}{2}+\alpha} w\right|\left|A^{\frac{1}{4}+\frac{\alpha}{2}} u\right|\left|A^{\frac{1}{4}+\frac{\alpha}{2}} v\right|,
$$

the proof of which refers to [8, Lemma 4.1]. Therefore,

$$
\begin{aligned}
& \left|A^{\alpha} Z_{t}^{\gamma}\right|^{2}+2 \int_{t_{0}}^{t}\left|A^{\alpha+\frac{1}{2}} Z_{s}^{\gamma}\right|^{2} d s \\
& \leq\left|A^{\alpha} Z_{t_{0}}^{\gamma}\right|^{2}+C_{1} \int_{t_{0}}^{t}\left|A^{\alpha+\frac{1}{2}} Z_{s}^{\gamma}\right|\left|A^{\frac{\alpha}{2}+\frac{1}{4}}\left(Z_{s}^{\gamma}+z_{s}^{\gamma}\right)\right|^{2} d s \\
& \quad+C_{1} \int_{t_{0}}^{t}\left(\left|A^{\alpha} Z_{s}^{\gamma}\right|^{2}+\left|A^{\alpha} Z_{s}^{\gamma}\right|\left|A^{\alpha} z_{s}^{\gamma}\right|\right) d s+\tilde{M}_{t} \\
& \leq\left|A^{\alpha} Z_{t_{0}}^{\gamma}\right|^{2}+C_{2} \int_{t_{0}}^{t}\left[\left|A^{\alpha+\frac{1}{2}} Z_{s}^{\gamma}\right|\left|A^{\frac{\alpha}{2}+\frac{1}{4}} Z_{s}^{\gamma}\right|^{2}+\left|A^{\alpha+\frac{1}{2}} Z_{s}^{\gamma}\right|\left|A^{\frac{\alpha}{2}+\frac{1}{4}} z_{s}^{\gamma}\right|^{2}\right. \\
& \left.\quad+\left|A^{\alpha} Z_{s}^{\gamma}\right|^{2}+\left|A^{\alpha} z_{s}^{\gamma}\right|^{2}\right] d s+\tilde{M}_{t} \\
& \leq\left|A^{\alpha} Z_{t_{0}}^{\gamma}\right|^{2}+C_{3} \int_{t_{0}}^{t}\left[\left|A^{\alpha} Z_{s}^{\gamma}\right|^{4 \alpha}\left|A^{\alpha+\frac{1}{2}} Z_{s}^{\gamma}\right|^{3-4 \alpha}+\left|A^{\alpha+\frac{1}{2}} Z_{s}^{\gamma}\right|\left|A^{\frac{\alpha}{2}+\frac{1}{4}} z_{s}^{\gamma}\right|^{2}\right.
\end{aligned}
$$




$$
\begin{gathered}
\left.+\left|A^{\alpha} Z_{s}^{\gamma}\right|^{2}+\left|A^{\alpha} z_{s}^{\gamma}\right|^{2}\right] d s+\tilde{M}_{t} \\
\leq\left|A^{\alpha} h\right|^{2}+\int_{t_{0}}^{t}\left|A^{\alpha+\frac{1}{2}} Z_{s}^{\gamma}\right|^{2} d s+C_{4} \int_{t_{0}}^{t}\left|A^{\alpha} Z_{s}^{\gamma}\right|^{2} d s \\
+C_{4} \int_{t_{0}}^{t}\left(\left|A^{\alpha} z_{s}^{\gamma}\right|^{2}+\left|A^{\frac{\alpha}{2}+\frac{1}{4}} z_{s}^{\gamma}\right|^{2}\right) d s+\tilde{M}_{t} .
\end{gathered}
$$

Hence

$$
\begin{aligned}
\mathbf{E} \sup _{s \leq} & \left|A^{\alpha} Z_{s}^{\gamma}\right|^{2}+\mathbf{E} \int_{t_{0}}^{t}\left|A^{\alpha+\frac{1}{2}} Z_{s}^{\gamma}\right|^{2} d s \\
\leq & \left|A^{\alpha} Z_{t_{0}}^{\gamma}\right|^{2}+C_{4} \mathbf{E} \int_{t_{0}}^{t}\left|A^{\alpha} Z_{s}^{\gamma}\right|^{2} d s+C_{4} \mathbf{E} \int_{t_{0}}^{t}\left(\left|A^{\alpha} z_{s}^{\gamma}\right|^{2}+\left|A^{\frac{\alpha}{2}+\frac{1}{4}} z_{s}^{\gamma}\right|^{2}\right) d s \\
& +\mathbf{E} \int_{t_{0}}^{t} \int_{U}\left(2 A^{\alpha} Z_{s-}^{\gamma} \cdot A^{\alpha}, F\left(Z_{s-}^{\gamma}+z_{s-}^{\gamma}, u\right)\right) \tilde{N}(d s, d u) \\
& +\mathbf{E} \int_{t_{0}}^{t} \int_{U}\left(\left|A^{\alpha}\left(Z_{s-}^{\gamma}+F\left(Z_{s-}^{\gamma}+z_{s-}^{\gamma}, u\right)\right)\right|^{2}-\left|A^{\alpha} Z_{s-}^{\gamma}\right|^{2}\right) N(d s, d u) \\
& -\mathbf{E} \int_{t_{0}}^{t} \int_{U}\left(2 A^{\alpha} Z_{s-}^{\gamma} \cdot A^{\alpha}, F\left(Z_{s-}^{\gamma}+z_{s-}^{\gamma}, u\right)\right) N(d s, d u) \\
\leq & \left|A^{\alpha} Z_{t_{0}}^{\gamma}\right|^{2}+C_{4} \mathbf{E} \int_{t_{0}}^{t}\left|A^{\alpha} Z_{s}^{\gamma}\right|^{2} d s+C_{4} \mathbf{E} \int_{t_{0}}^{t}\left(\left|A^{\alpha} z_{s}^{\gamma}\right|^{2}+\left|A^{\frac{\alpha}{2}+\frac{1}{4}} z_{s}^{\gamma}\right|^{2}\right) d s \\
& +\mathbf{E} \int_{t_{0}}^{t} \int_{U}\left|\left(2 A^{\alpha} Z_{s-}^{\gamma} \cdot A^{\alpha}, F\left(Z_{s-}^{\gamma}+z_{s-}^{\gamma}, u\right)\right)\right| \tilde{N}(d s, d u) \\
& +\mathbf{E} \int_{t_{0}}^{t} \int_{U} \mid \int_{0}^{1}\left(2 A^{\alpha}\left(Z_{-s}+s^{\prime} F\left(Z_{s-}^{\gamma}+z_{s-}^{\gamma}, u\right)\right) \cdot A^{\alpha},\right. \\
& \left.F\left(Z_{s-}^{\gamma}+z_{s-}^{\gamma}, u\right)\right) d s^{\prime} \mid N(d s, d u) \\
& +C_{6} \mathbf{E} \int_{t_{0}}^{t}\left(\left|A^{\alpha} z_{s}^{\gamma}\right|^{2}+\left|A^{\frac{\alpha}{2}+\frac{1}{4}} z_{s}^{\gamma}\right|^{2}\right) d s, \\
& +\mathbf{E} \int_{t_{0}}^{t} \int_{U}\left|\left(2 A^{\alpha} Z_{s-}^{\gamma} \cdot A^{\alpha}, F\left(Z_{s-}^{\gamma}+z_{s-}^{\gamma}, u\right)\right)\right| N(d s, d u) \\
\leq & \left|A^{\alpha} Z_{t_{0}}^{\gamma}\right|^{2}+C_{t_{0}}{ }^{2} \mathbf{E} \int_{t_{0}}^{t}\left|A^{\alpha} Z_{s}^{\gamma}\right|^{2} d s+C_{4} \mathbf{E} \int_{t_{0}}^{t}\left(\left|A^{\alpha} z_{s}^{\gamma}\right|^{2}+\left|A^{\frac{\alpha}{2}+\frac{1}{4}} z_{s}^{\gamma}\right|^{2}\right) d s \\
& +C_{5}\left[\mathbf{E} \int_{t_{0}}^{t} \int_{U}\left|A^{\alpha} Z_{s}^{\gamma}\right|^{2} \cdot\left|A^{\alpha} F\left(Z_{s}^{\gamma}+z_{s}^{\gamma}, u\right)\right|^{2} \lambda(d u) d s\right]^{\frac{1}{2}} \\
& \\
& \\
&
\end{aligned}
$$

where $\varepsilon \in(0,1)$ and the last inequality is derived by combining hypotheses $\left(\mathrm{H}_{2}\right),\left(\mathrm{H}_{3}\right)$ with the Young inequality. 
Henceforth,

$$
\begin{aligned}
& (1-\varepsilon) \mathbf{E} \sup _{s \leq t}\left|A^{\alpha} Z_{s}^{\gamma}\right|^{2}+\mathbf{E} \int_{t_{0}}^{t}\left|A^{\alpha+\frac{1}{2}} Z_{s}^{\gamma}\right|^{2} d s \\
& \quad \leq\left|A^{\alpha} Z_{t_{0}}^{\gamma}\right|^{2}+C_{6} \mathbf{E} \int_{t_{0}}^{t}\left|A^{\alpha} Z_{s}^{\gamma}\right|^{2} d s+C_{6} \mathbf{E} \int_{t_{0}}^{t}\left(\left|A^{\alpha} z_{s}^{\gamma}\right|^{2}+\left|A^{\frac{\alpha}{2}+\frac{1}{4}} z_{s}^{\gamma}\right|^{2}\right) d s .
\end{aligned}
$$

If $t_{0} \leq-1$

$$
\begin{aligned}
\mathbf{E}\left|Z^{\gamma}\left(0, t_{0}\right)\right|^{2} & \leq e^{2 C_{6}}\left[\left|A^{\alpha} Z^{\gamma}\left(-1, t_{0}\right)\right|^{2}+C_{6} \mathbf{E} \int_{-1}^{0}\left(\left|A^{\alpha} z_{s}^{\gamma}\right|^{2}+\left|A^{\frac{\alpha}{2}+\frac{1}{4}} z_{s}^{\gamma}\right|^{2}\right) d s\right] \\
& \leq e^{2 C_{7}}\left[\left|A^{\frac{1}{2}} Z^{\gamma}\left(-1, t_{0}\right)\right|^{2}+C_{6} \mathbf{E} \int_{-1}^{0}\left(\left|A^{\alpha} z_{s}^{\gamma}\right|^{2}+\left|A^{\frac{\alpha}{2}+\frac{1}{4}} z_{s}^{\gamma}\right|^{2}\right) d s\right] .
\end{aligned}
$$

Repeating the argument similarly in Lemma 3.1, $\left(\mathrm{H}_{1}\right)$ implies that $z_{t}^{\gamma}$ has a continuous version in $\mathscr{D}\left(A^{\frac{1}{4}+\frac{\alpha}{2}}\right)$, and therefore, also has a continuous version in $\mathscr{D}\left(A^{\alpha}\right)$. Furthermore, E $\int_{-1}^{0}\left(\left|A^{\alpha} z_{s}^{\gamma}\right|^{2}+\left|A^{\frac{\alpha}{2}+\frac{1}{4}} z_{s}^{\gamma}\right|^{2}\right) d s$ is finite. Following almost the same argument as [4, Proposition15.4.3], it can be proved that there exists $\gamma>0$ such that $\left|A^{\frac{1}{2}} Z^{\gamma}\left(-1, t_{0}\right)\right|^{2}$ is finite a.e. for $t_{0} \leq-1$.

Therefore, both $\sup _{t_{0} \leq-1} \mathbf{E}\left|A^{\alpha} Z^{\gamma}\left(0, t_{0}\right)\right|^{2}$ and $\sup _{t_{0} \leq-1} \mathbf{E}\left|A^{\alpha} X\left(0, t_{0}\right)\right|^{2}$ are finite. Following the classical arguments (cf. [12]), we can prove the tightness of $\left\{\mu_{T}(\cdot)=\frac{1}{T} \int_{-T}^{0} P(s, x, \cdot), T>\right.$ $0\}$, which implies the existence of an invariant measure $\mu$.

\section{Discussion}

In this section, we will generalize the result of well-posedness for stochastic Boussinesq equation (2.1) to some of the stochastic hydrodynamical systems, such as 2D-stochastic Navier-Stokes equation, 2D magneto-hydrodynamic equation, 2D Boussinesq model for the Bénard convection, 2D magnetic Bénard problem, and so on. We adopt the notations and assumptions proposed in [2] and refer to [11, 14, 21, 22] for more examples.

We will introduce an abstract framework for the stochastic hydrodynamical systems. Let $H$ be a separable Hilbert space with the norm $|\cdot|$, and the operator $A$ be an unbounded selfadjoint positive linear operator on $H$. Denote $V=\operatorname{Dom}\left(A^{\frac{1}{2}}\right)$ with the norm $\|v\|=\left|A^{\frac{1}{2}} \nu\right|$. Let $V^{\prime}$ be the dual of $V$. For any $u \in V, v \in V^{\prime}$, the duality product between $V$ and $V^{\prime}$ is denoted by $\langle u, v\rangle$. Suppose that the mapping $B: V \times V \rightarrow V^{\prime}$ satisfies the following assumptions:

- (H(i)) $B(\cdot, \cdot): V \times V \rightarrow V^{\prime}$ is a continuous bilinear mapping.

- (H(ii)) For any $u_{i} \in V, i=1,2,3$, there holds

$$
\left\langle B\left(u_{1}, u_{2}\right), u_{3}\right\rangle=-\left\langle B\left(u_{1}, u_{3}\right), u_{2}\right\rangle .
$$

- (H(iii)) There exists a Banach space $\mathscr{H}$ which satisfies

(iii-1) $V \subset \mathscr{H} \subset H$;

(iii-2) there exists a positive constant $\alpha_{0}$ such that

$$
\|v\|_{\mathscr{H}}^{2} \leq \alpha_{0}|v| \cdot\|v\|, \quad \text { for any } v \in V
$$


(iii-3) for any $\eta>0$, there exists a positive constant $C_{\eta}$ such that

$$
\left|\left\langle B\left(u_{1}, u_{2}\right), u_{3}\right\rangle\right| \leq \eta\left\|u_{3}\right\|^{2}+C_{\eta}\left\|u_{1}\right\|_{\mathscr{H}}^{2} \cdot\left\|u_{2}\right\|_{\mathscr{H}}^{2}, \quad u_{i} \in V, i=1,2,3 .
$$

As we will see, the majority of stochastic hydrodynamical systems with Lévy noise, such as stochastic two-dimensional Navier-Stokes equation, stochastic two-dimensional Boussinesq equations, stochastic two-dimensional magnetic Bénard equations, and stochastic two-dimensional magneto-hydrodynamic equations, can be represented uniformly as the following stochastic evolution equation (see [20]):

$$
\left\{\begin{array}{c}
d u(t)+[A u(t)+B(u(t), u(t))+R(u(t))] d t \\
=Q d W(t)+\int_{X} F(u(t-), x) \tilde{N}(d t, d x), \\
u(0)=u_{0},
\end{array}\right.
$$

where $R(\cdot)$ and $Q$ are linear bounded operators in $H, W(\cdot)$ is an $H$-valued Brownian motion, $F$ is a measurable mapping from some measurable space $X$ to $H, \tilde{N}$ is a compensated Poisson measure on $[0, \infty) \times X$ with intensity measure $v$. Additionally, we need

- (H(iv)) There exist positive constants $C$ and $K$ such that, for some $\alpha \in[1 / 4,1 / 2)$,

- (H(iv-1)) $v$ is a $\sigma$-finite measure on $\mathscr{B}(X), Q: H \rightarrow H$ is a linear bounded operator with range $\mathscr{R}(Q)$ dense in $\mathscr{D}\left(A^{\frac{1}{4}+\frac{\alpha}{2}}\right)$ and $\mathscr{D}\left(A^{2 \alpha}\right) \subset \mathscr{R}(Q) \subset \mathscr{D}\left(A^{\frac{1}{4}+\frac{\alpha}{2}+\varepsilon}\right)$ for some $\varepsilon>0$;

- $(\mathrm{H}(\mathrm{iv}-2)) \int_{U}\left|A^{\alpha} F(0, u)\right|^{2} \lambda(d u)=C$;

- $(\mathrm{H}(\mathrm{iv}-3)) \int_{U}\left|A^{\alpha}(F(x, u)-F(y, u))\right|^{2} \lambda(d u) \leq K\left|A^{\alpha}(x-y)\right|^{2}$;

- (H(iv-4)) $\sup _{x \in H} \int_{U_{m}^{c}}\left|A^{\alpha} F(x, u)\right|^{2} \lambda(d u) \rightarrow 0$, as $m \rightarrow \infty$.

Repeating similar arguments within Sect. 3 and Sect. 4 with minor modification, we can obtain the following result.

Theorem 5.1 Assume that hypotheses $\mathrm{H}(\mathrm{i})-\mathrm{H}(\mathrm{iv})$ hold. For every $u_{0} \in \mathscr{D}\left(A^{\alpha}\right)$, there exists a unique fragile solution $u$ to equation (5.4). Denote by $P_{t}$ the Markov semigroup on $H$ generated by the solution flow. Then $P_{t}$ is a Feller Markov process. Furthermore, there exists at least one invariant measure $\mu$ for the transition semigroup $P_{t}$.

Acknowledgements

The authors would like to thank the editor and the reviewers for giving us constructive suggestions which would help us both in English and in depth to improve the quality of the paper.

\section{Funding}

This work was supported by the National Natural Science Foundation of China (No. 11771449), NSF of Hunan (No. 2018JJ2468), and the Fundamental Program of NUDT (No. ZK17-03-19).

\section{Competing interests}

The authors declare to have no competing interests.

Authors' contributions

All authors participated in drafting and checking the manuscript and approved the final manuscript.

\section{Publisher's Note}

Springer Nature remains neutral with regard to jurisdictional claims in published maps and institutional affiliations. 


\section{References}

1. Brune, P., Duan, J., Schmalfuss, B.: Random dynamics of the Boussinesq with dynamical boundary conditions. Stoch. Anal. Appl. 27(5), 1096-1116 (2009)

2. Chueshov, I., Millet, A.: Strochastic 2D hydrodynamical type systems: well posedness and large deviations. Appl. Math. Optim. 61(3), 379-420 (2010)

3. Constantin, P., Foias, C.: Navier-Stokes Equations. University of Chicago Press, Chicago (1988)

4. Da Prato, G., Zabczyk, J.: Ergodicity for Infinite Dimensional Systems. Cambridge University Press, Cambridge (1996)

5. Dong, Z.: The uniqueness of invariant measure of the Burgers equation driven by Levy processes. J. Theor. Probab. 21(2), 322-335 (2008)

6. Dong, Z., Xie, Y.: Global solutions of stochastic 2D Navier-Stokes equations with Levy noise. Sci. China Ser. A, Math. 52(7), 1497-1524 (2009)

7. Duan, J., Millet, A.: Large deviations for the Boussinesq equations under random influences. Stoch. Process. Appl. 119, 2052-2081 (2009)

8. Flandoli, F.: Dissipativity and invariant measures for stochastic Navier-Stokes equation. NoDEA Nonlinear Differ. Equ. Appl. 1, 403-423 (1994)

9. Foias, C., Manley, O., Temam, R.: Attractors for the Benard problem: existence and physical bounds on their fractal dimension. Nonlinear Anal. 11, 939-967 (1987)

10. Földes, J., Glatt-Holtz, N., Richards, G., et al.: Ergodic and mixing properties of the Boussinesq equations with a degenerate random forcing. J. Funct. Anal. 269(8), 2427-2504 (2015)

11. Huang, J., Shen, T.: Well-posedness and dynamics of the stochastic fractional magneto-hydrodynamic equations. Nonlinear Anal., Theory Methods Appl. 133, 102-133 (2016)

12. Peszat, S., Zabczyk, J.: Stochastic Partial Differential Equations with Levy Noises-Evolution Equation Approach. Cambridge University Press, Cambridge (2009)

13. Shen, T., Huang, J.: Well-posedness of the stochastic fractional Boussinesq equation with Levy noise. Stoch. Anal. Appl. 33(6), 1092-1114 (2015)

14. Shen, T., Huang, J.: Ergodicity of stochastic magneto-hydrodynamic equations driven by alpha-stable noise. J. Math. Anal. Appl. 446(1), 746-769 (2017)

15. Shen, T., Huang, J., Li, J.: Stochastic modified Boussinesq approximate equation driven by fractional Brownian motion. Adv. Differ. Equ. 2014(1), 207 (2014)

16. Sun, F., Gao, H., Duan, J., Schmalfuss, B.: Rare events in the Boussinesq system with fluctuating dynamical boundary conditions. J. Differ. Equ. 248, 1269-1296 (2010)

17. Teman, R.: Infinite-Dimensional Dynamical Systems in Mechanics and Physics. Springer, New York (1988)

18. Yamazaki, K.: Global martingale solution for the stochastic Boussinesq system with zero dissipation. Stoch. Anal. Appl. 34(3), 404-426 (2016)

19. Zhang, $Y .: H^{1}$-Random attractors and asymptotic smoothing effect of solutions for stochastic Boussinesq equations with fluctuating dynamical boundary conditions. Abstr. Appl. Anal. 2013, Article ID 706091 (2013)

20. Zheng, Y., Huang, J.: Large deviation principle for stochastic Boussinesq equations driven by Levy noise. J. Math. Anal. Appl. 439(2), 523-550 (2016)

21. Zou, G.A., Lv, G., Wu, J.L.: Stochastic Navier-Stokes equations with Caputo derivative driven by fractional noises J. Math. Anal. Appl. 461(1), 595-609 (2018)

22. Zou, G.A., Wang, B.: Stochastic Burgers' equation with fractional derivative driven by multiplicative noise. Comput. Math. Appl. 74, 3195-3208 (2017)

\section{Submit your manuscript to a SpringerOpen ${ }^{\circ}$ journal and benefit from:}

- Convenient online submission

- Rigorous peer review

- Open access: articles freely available online

- High visibility within the field

- Retaining the copyright to your article

Submit your next manuscript at $>$ springeropen.com 